\title{
Central metabolic responses of microorganisms to years and decades of soil warming
}

\author{
Andrea Söllinger ( $\square$ andrea.soellinger@uit.no ) \\ Uit - The Arctic University of Norway \\ Mathilde Borg Dahl \\ University of Greifswald \\ Joana Séneca \\ University of Vienna \\ Judith Prommer \\ University of Vienna

\section{Erik Verbruggen} \\ University of Antwerp https://orcid.org/0000-0001-7015-1515

\section{Bjarni Sigurdsson}

Agricultural University of Iceland https://orcid.org/0000-0002-4784-5233

Ivan Janssens

University of Antwerp https://orcid.org/0000-0002-5705-1787

\section{Josep Penuelas}

CSIC, Global Ecology Unit CREAF-CSIC-UAB, Cerdanyola del Vallès 08193, Catalonia, Spain

https://orcid.org/0000-0002-7215-0150

\section{Tim Urich}

Institute of Microbiology, University of Greifswald

\section{Andreas Richter}

University of Vienna https://orcid.org/0000-0003-3282-4808

\section{Alexander Tveit}

UiT, The Arctic University of Norway

\section{Article}

Keywords: metatranscriptomics, microorganisms, soil warming, microbial biomass

Posted Date: February 5th, 2021

DOI: https://doi.org/10.21203/rs.3.rs-132190/v1

License: (1) (1) This work is licensed under a Creative Commons Attribution 4.0 International License. 



\section{Central metabolic responses of microorganisms to years and}

\section{2 decades of soil warming}

3

4 Andrea Söllinger ${ }^{1^{*}}$, Mathilde Borg Dahl ${ }^{2}$, Joana Séneca ${ }^{3}$, Judith Prommer ${ }^{3}$, Erik Verbruggen ${ }^{4}$, Bjarni D.

5 Sigurdsson ${ }^{5}$, Ivan Janssens ${ }^{4}$, Josep Peñuelas ${ }^{6,7}$, Tim Urich $^{2}$, Andreas Richter ${ }^{3}$, Alexander T. Tveit ${ }^{1^{*}}$

6

$7{ }^{1}$ Department of Arctic and Marine Biology, UiT The Arctic University of Norway, Troms $\varnothing$, Norway

$8 \quad{ }^{2}$ Institute of Microbiology, University of Greifswald, Greifswald, Germany

$9{ }^{3}$ Centre for Microbiology and Environmental Systems Science, University of Vienna, Vienna, Austria

${ }^{4}$ PLECO, University of Antwerp, Antwerp, Belgium

$11{ }^{5}$ Agricultural University of Iceland, Borgarnes, Iceland

$12{ }^{6}$ CSIC, Global Ecology Unit CREAF-CSIC-UAB, Barcelona, Spain

$13{ }^{7}$ CREAF, Barcelona, Spain

14

$15 *$ Corresponding authors 
Microbial physiological responses to long-term warming are poorly understood. Here we applied metatranscriptomics to investigate how microorganisms react to medium-term ( 8 years) and longterm ( $>5$ decades) subarctic grassland soil warming of $+6^{\circ} \mathrm{C}$.

Decades, but not years, of warming induced changes in relative abundances of eukaryotic, prokaryotic, and viral transcripts and reduced functional richness. However, irrespective of the duration of warming, we observed a community-wide upregulation of central (carbon) metabolisms and cell replication in the warmed soils, whereas essential energy metabolism and protein biosynthesis complexes and pathways were downregulated. This coincided with a decrease of microbial biomass and lower soil substrate concentrations (e.g. dissolved organic carbon and phosphorus). We conclude that permanently accelerated reaction rates at higher temperatures facilitate a downregulation of energy metabolism and protein biosynthesis, potentially freeing energy and matter for substrate acquisition and growth. This resource allocation seems to be a common response in microorganisms and allows sustaining high metabolic activities and replication rates even after decades of soil warming. 


\section{Introduction}

Global temperatures and atmospheric carbon dioxide $\left(\mathrm{CO}_{2}\right)$ levels have increased steadily over the last 100 years ${ }^{1,2}$. The terrestrial carbon (C) cycle feedback to the climate system represents a major uncertainty in the prediction of future temperatures ${ }^{3}$. Soil microorganisms, including Bacteria, Archaea, Fungi, and protists, are responsible for the turnover of soil organic matter (SOM) and the subsequent release of $\mathrm{CO}_{2}$ from soils to the atmosphere ${ }^{2}$. Higher temperatures commonly lead to higher microbial activities, so global warming should accelerate the decomposition of SOM to $\mathrm{CO}_{2}{ }^{4}$. On the other hand, SOM consists largely of microbial necromass and warming may stimulate microbial growth and thus necromass production ${ }^{5,6}$, promoting SOM formation. Whether soils will ultimately act as $\mathrm{C}$ sinks or sources, thus depend on how microorganisms respond to long-term warming. Nevertheless, microbial responses to global warming are currently poorly represented in Earth system models ${ }^{7}$, which can, to some extent, be attributed to the challenges associated with studying and quantifying microbial responses to environmental change in complex soil environments ${ }^{4}$.

Microbial responses to long-term soil warming may include i) quantitative and compositional changes of microbial communities, ii) physiological adjustments of individual microorganisms, including changes in growth and resource use, via transcriptional and translational regulation, iii) shifts in microbial interactions and emergent properties of the community, and iv) microbial adaptations by genomic rearrangements and evolutionary changes of the genetic code.

In one of the few truly long-term warming studies (Harvard Forest Warming Experiment: $26 \mathrm{y},+5^{\circ} \mathrm{C}$, mid-latitude forest soil $)^{8}$, the observed warming effects on the microbial community included a decrease in fungal biomarkers and abundance, a decrease in microbial biomass, a community shift toward Gram-positive Bacteria, and an increase in bacterial evenness and abundance of Bacteria with low copy numbers of ribosomal RNA (rRNA) operons. A meta-analysis of 25 in situ soil warming experiments ( $1-15 \mathrm{y},+0.5-5.5^{\circ} \mathrm{C}$, various soil ecosystems) found initial increases in soil respiration 
(46 $\pm 8 \%$ ) due to warming followed by significant decreases over time ${ }^{9}$. However, less than half of the studies estimated microbial biomass, and two thirds of the studies that quantified changes reported decreases in microbial biomass with warming. Increased rates of SOM degradation and soil respiration followed by a return to pre-warming rates within a few years were repeatedly observed ${ }^{10}$. This pattern is often explained by an "acclimation of soil respiration"; a shift in the response of respiration to ongoing warming that leads to different sensitivities of soil respiration to temperature ${ }^{11,12}$ (and references therein). Alternatively, a return to pre-warming states can be explained by the depletion of easily degradable substrates ${ }^{10,13}$.

It has recently been shown that natural geothermal activity can enable the study of soil warming on decadal time scales ${ }^{14,15}$. We here make use of the longest in situ soil warming experiment worldwide, ForHot, in which a subarctic grassland site has been exposed to soil warming for decades $(>50 \mathrm{y})^{14}$. More recent soil temperature gradients emerged nearby after an earthquake in 2008 . The effects of long-term and short- to medium-term soil warming on abiotic and biotic properties and processes at these sites have been described in a range of publications. For example, Walker et $a l .{ }^{16}$, MarañónJiménez et $a l .{ }^{17,18}$, and Poeplau et al. ${ }^{19}$ identified considerable soil environmental changes in the warmed grassland plots, including reductions in topsoil C and nitrogen $(\mathrm{N})$ pools by about $40 \%$ and decreased soil aggregation. These changes were accompanied by lower soil microbial biomass but higher soil respiration rates per unit of microbial biomass ${ }^{16}$, contradicting the concept of a physiological acclimation of microorganisms ${ }^{16}$. Amplicon sequencing of rRNA genes, on the other hand, did not indicate warming-induced changes of the microbial community composition at genus and operational taxonomic unit (OTU) level ${ }^{15,16,20}$. Recently a meta-analysis on 128 measured variables at this site, including a broad variety of biotic and abiotic soil properties, pools, and processes was published, reporting a systemic overreaction to 5-8 years versus decades of warming ${ }^{15}$. However, details of underlying microbial responses influencing the ecosystem-scaled responses remained largely unexplored. 
Here we aimed at providing the first in-depth functional analysis of soil microbial responses to warming using a metatranscriptomics approach, i.e. high-throughput shotgun-sequencing of total RNA. In contrast to DNA based methods (e.g., metagenomics or amplicon sequencing), which can only show genetic potential and are often restricted to specific microbial groups, metatranscriptomics allows the comprehensive study of the soil microbiome, i.e., the entire active soil microbial community (Bacteria, Archaea, Eukaryotes, and viruses) and their functions, by studying expressed genes (messenger RNA (mRNA) and rRNA) ${ }^{21}$. We analysed 16 soil microbiomes. In situ microbial gene expression profiles of long-term warmed soils (LTW) exposed to $+6{ }^{\circ} \mathrm{C}$ above ambient $\left(E_{T}\right)$ for $>50$ y and medium-term warmed soils (MTW) exposed to $+6{ }^{\circ} \mathrm{C}$ for 8 y were compared to ambient controls $\left(\mathrm{A}_{\mathrm{T}}\right)$. Additionally, we measured dissolved and total soil $\mathrm{C}, \mathrm{N}$ and phosphorous $(\mathrm{P})$ concentrations and estimated microbial biomass. Our main objective was to elucidate if and how soil microorganisms alter their cellular activities and functions in response to warming.

\section{Results}

\section{Warming effects on soil physicochemical and biological properties}

Warmed soils differed from their ambient counterparts (Fig. 1b); total, dissolved organic, and microbial C, N, and P concentrations were lower in the warmed soils, mirroring previous studies that also report lower substrate concentrations and microbial biomass contents in the warmed soils at the same site ${ }^{15-18}$. Soil pH ranged from 4.4 to 6.0 and was slightly higher in the warmed soils $\left(P<0.05, \mathrm{n}=16, P_{\text {corr }}>0.1\right.$, Supplementary Table 1, 2). 

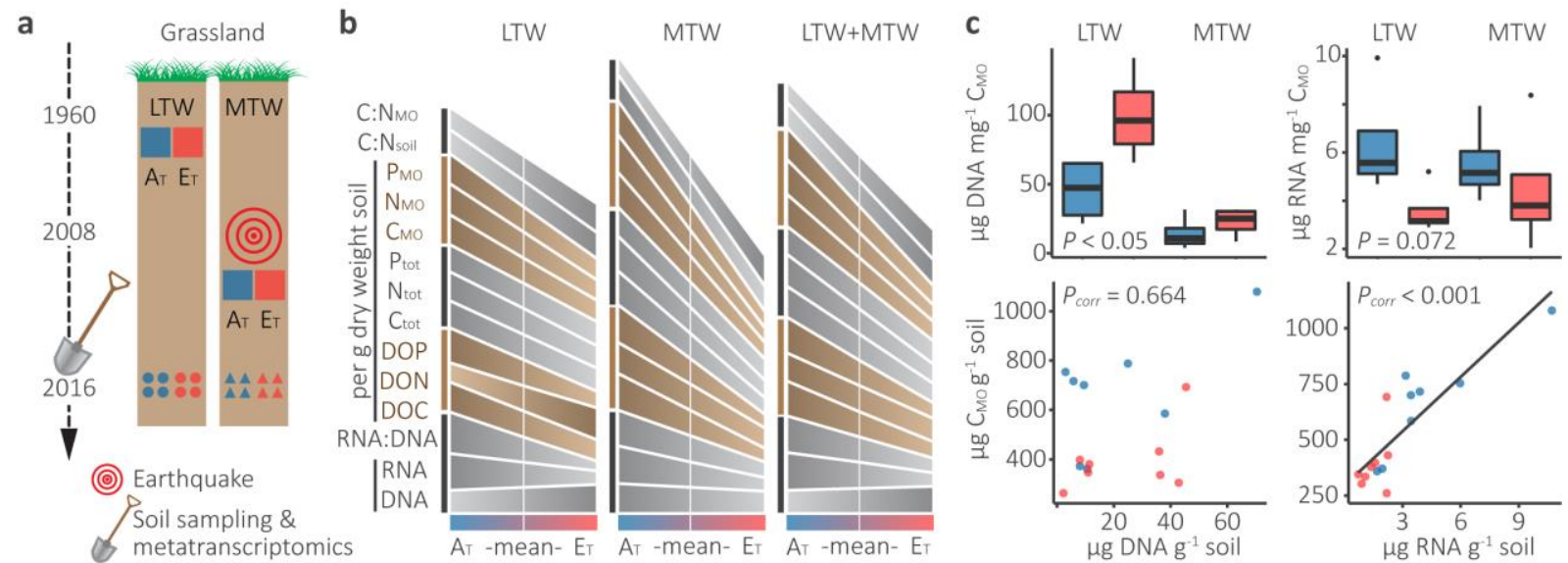

Figure 1. Grassland samples and warming-induced differences on physicochemical and biological properties. a Schematic

representation of the study sites, their development over time, and the metatranscriptomics samples (see Methods for

details). b Trend charts (see Methods) indicating differences in DNA and RNA concentrations (per unit of soil), contents of

dissolved organic $\mathrm{C}, \mathrm{N}$, and $\mathrm{P}$ (DOC, DON, and DOP, respectively), total $\mathrm{C}, \mathrm{N}$, and $\mathrm{P}\left(\mathrm{C}_{\text {tot }}, \mathrm{N}_{\text {tot }}\right.$, and $\mathrm{P}_{\text {tot }}$, respectively), microbial

$C, N$, and $P\left(C_{M O}, N_{M O}\right.$, and $P_{M O}$, respectively), RNA:DNA ratio, and soil and microbial C:N ratios (see Supplementary Tables 1 and 2 for absolute values and significant differences, respectively). c DNA and RNA content per unit of microbial biomass and correlations between microbial biomass and DNA and RNA content per unit of soil (Supplementary Table 3).

DNA and RNA concentrations per unit of microbial $\mathrm{C}\left(\mathrm{C}_{\mathrm{MO}}\right)$ had opposite trends, with higher DNA but lower RNA concentrations in the warmed soils, irrespective of the warming duration (Fig. 1c). While the RNA content per $g$ soil positively correlated with $\mathrm{C}_{\mathrm{MO}}\left(r_{\mathrm{s}(16)}=0.81, P_{\text {corr }}<0.001\right)$, the DNA content did not $\left(r_{\mathrm{s}(16)}=0.12, P_{\text {corr }}=0.664\right)$ (Fig. $\left.1 \mathrm{c}\right) . \mathrm{C}_{\mathrm{MO}}$ was likewise positively correlated with total soil $\mathrm{C}, \mathrm{N}$, and $\mathrm{P}$, dissolved organic $\mathrm{C}$ and $\mathrm{P}$, microbial $\mathrm{N}$ and $\mathrm{P}$, and water content (Supplementary Table 3 ).

To investigate what structural and functional changes in the soil microbiomes were associated with these substantial differences in microbial biomass and soil chemistry we performed metatranscriptomics.

\section{Compositional changes of the functional microbial communities across all domains of life}

Illumina paired-end sequencing produced an average of $6.69 \times 10^{6} \mathrm{mRNA}$ reads per sample

123 (Supplementary Table 4). Bacteria dominated the mRNA transcript pools (93.36-98.52\%), followed 
different families were detected, and mean family richness was lower in the heated soils, albeit not significant (Supplementary Fig. 1a). An NMDS analysis of mRNA reads assigned at family level indicated no clear overall separation between $A_{T}$ and $E_{T}$ samples and a greater variability between MTW replicates than LTW replicates (Supplementary Fig. 2a). Thus, we analysed the detailed taxonomic assignments of LTW and MTW separately (Fig. 2). In the long-term warmed soils, but not in the medium-term warmed soils, several taxa showed warming-induced differences in relative transcript abundances (Fig. 2a, Supplementary Table 6). Those that may be affected by warming include phylogenetic groups across all domains of life and included Fungi, protists, and Chloroflexi, which showed lower mean relative abundances in $\mathrm{LTW}-\mathrm{E}_{\mathrm{T}}$, and Deltaproteobacteria, Planctomycetes, Verrucomicrobia, as well as viruses, which showed higher mean relative abundances in LTW- $\mathrm{E}_{\mathrm{T}}$ compared to LTW-A (Fig. 2a, b). A taxonomic analysis of rRNA reads corroborated the bacterial mRNA profiles, but the mean relative abundances of Eukaryotes differed between in the rRNA datasets and the mRNA datasets (Supplementary Fig. 3).

We further screened all taxonomic ranks down to family level (Fig. 2c). Five taxa showed lower relative expression levels in $L T W-E_{T}$ than $L T W-A_{T}$, and 17 taxa showed higher relative expression levels in LTW- $E_{T}$ than LTW-A $($ Fig. 2c). Differential gene expression analyses underpinned these results, as many of the reported taxa were significantly different. In contrast, none of the six taxa that displayed higher relative expression levels in MTW- $E_{T}$ than $M T W-A_{T}$ was significantly differential expressed (Fig. 2c). We repeated the analysis on the bacterial fraction of the mRNAs to test whether Eukaryota abundances influenced the bacterial patterns and found almost identical warming-induced abundance patterns (Supplementary Fig. 4). We also attempted to increase the taxonomic resolution by assembling the metatranscriptome reads (Supplementary Methods). Our approach using rnaSpades ${ }^{22}$, however, did not provide a sufficient number of long mRNA contigs $(<10 \%$ of functionally annotated mRNA contigs were longer than the unassembled reads). 
a

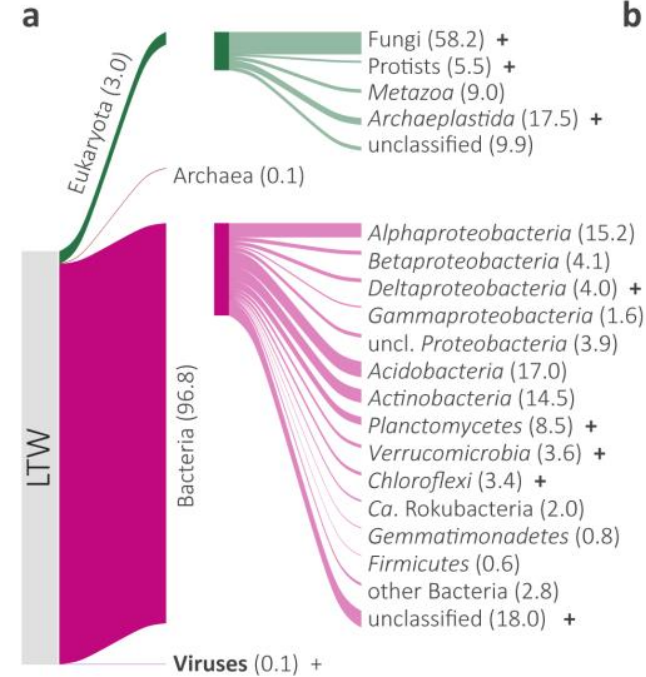

149

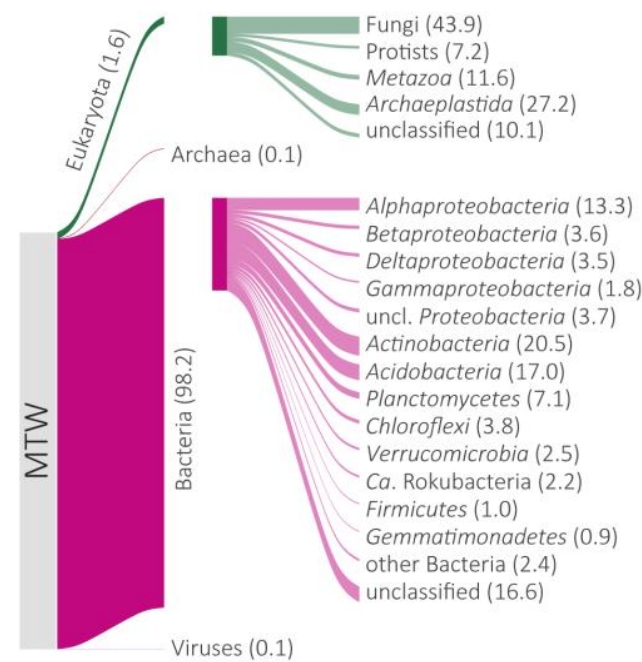

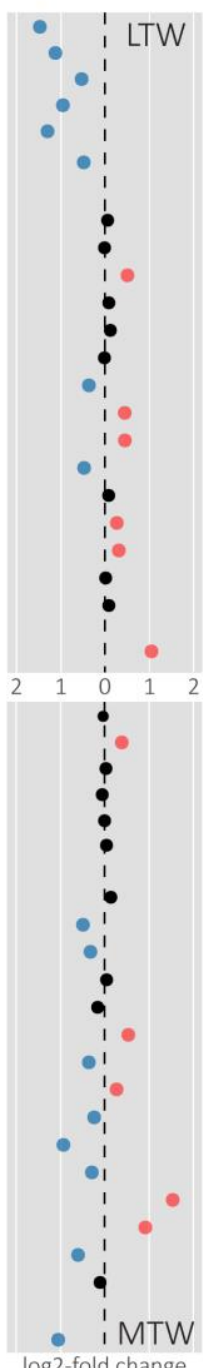

C

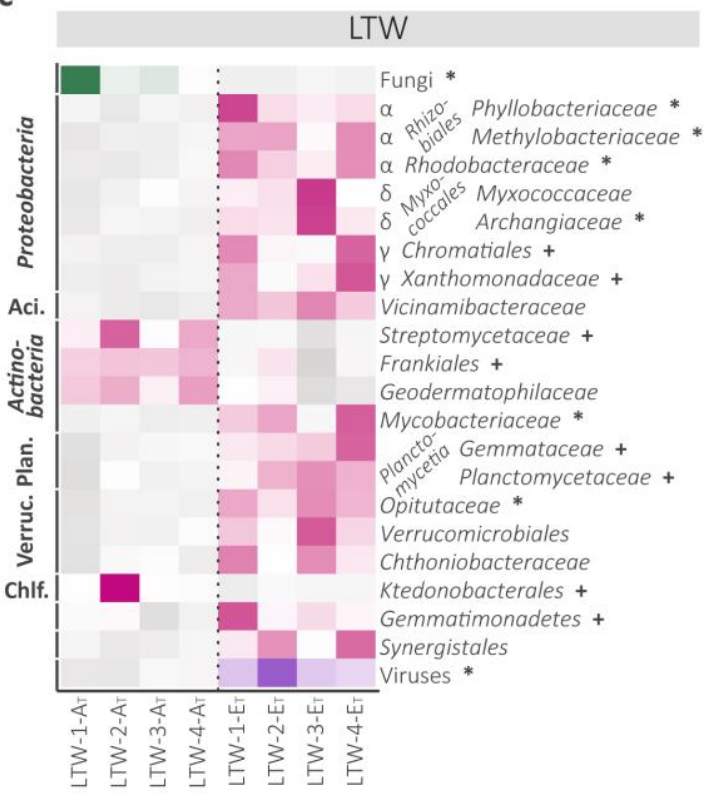

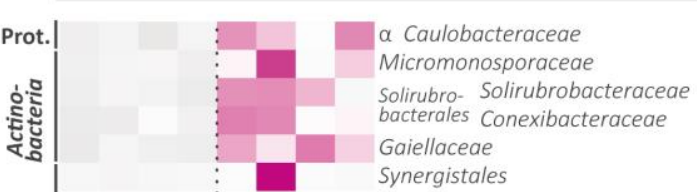

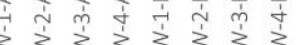

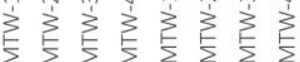
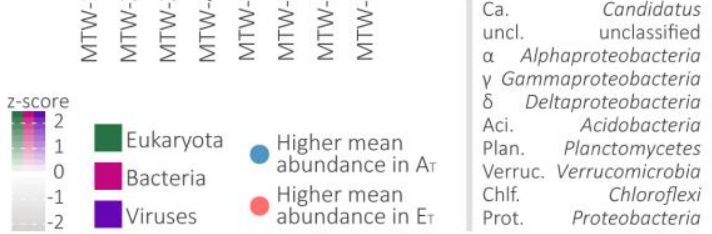

Fig. 2. Taxonomic annotation of mRNA reads and warming-induced abundance patterns. a Sankey plots showing both the

fraction of mRNA reads (mean over all LTW and MTW replicates, respectively) assigned to domains and the composition and relative abundances of eukaryotic and bacterial mRNAs. The depicted groups account for $100 \%$ of eukaryotic and bacterial mRNAs, respectively. All bacterial phyla with a relative abundance of $\geq 1 \%$ in at least one of the sampled soil groups are depicted; the remaining phyla are summed (other Bacteria). Potential warming-induced differences in mRNA abundances are indicated with + (t-tests, $\mathrm{n}=8, P<0.05, P_{\text {corr }}<0.1$; Supplementary Table 6). $\mathbf{b}$ Log2-fold changes of mean relative abundances between $A_{T}$ and $E_{T}$ of the taxa listed left-hand. $c$ Exploratory analysis showing warming-induced taxon abundance patterns. Only taxa with higher or lower relative abundances in all four replicates of one group relative to their counterparts are depicted (4/4-filter, see Methods). Bacterial taxa are shown at family level; higher taxonomic levels are only shown if no family belonging to these higher levels passed the filter. Higher bacterial taxonomic levels are accordingly not shown if any family belonging to these levels passed the filter. Potential warming-induced differences in mRNA abundances are indicated (differential gene expression analysis, $\mathrm{n}=8, P_{\text {corr }}<0.05\left({ }^{*}\right), P_{\text {corr }}<0.1(+)$; Supplementary Table 7). 
We subsequently analysed functional mRNA annotations to identify how soil warming influences the transcription of genes involved in central metabolic functions and cellular processes.

\section{Warming-induced gene expression profiles}

An average of $2.66 \times 10^{6}$ mRNA reads per sample (Supplementary Table 4) was assigned to a molecular function (KO number) defined in the KEGG Orthology database ${ }^{23}$. KEGG offers a hierarchical structure with four layers, hereafter referred to as KEGG1 (i.e. Metabolism, Genetic and Environmental information processing, and Cellular processes), KEGG2 (see Fig. 3), KEGG3 (i.e. pathways and functional complexes), and KO (molecular functions, i.e. enzymes and enzyme subunits). Significantly fewer unique functions (KOs) were detected in LTW-ET than LTW-AT (t-tests, $n$

$172=8, P<0.05)$, but not between MTW- $E_{T}$ and MTW-A $($ Supplementary Fig. 1b). PERMANOVA analyses

173 further revealed a significant effect of warming on expressed functions (KOs), and the functions expressed by different microbial families (Supplementary Fig. 2c, d; Supplementary Table 5).

To identify the nature of this functional response we explored in more detail the functional assignments to KEGG1 categories Metabolism (65.4\%), Genetic information processing (16.8\%), Cellular processes (9.0\%), and Environmental information processing (8.9\%) (Fig. 3). Transcripts for Metabolism and major Metabolism subcategories such as Carbohydrate metabolism, Lipid metabolism, and Nucleotide metabolism had higher relative abundances in the warmed soils, while Genetic information processing subcategories such as Transcription and Translation had lower relative abundances (Fig. 3, Supplementary Table 8). Transcript patterns for Cellular processes and Environmental information processing were not consistent. 


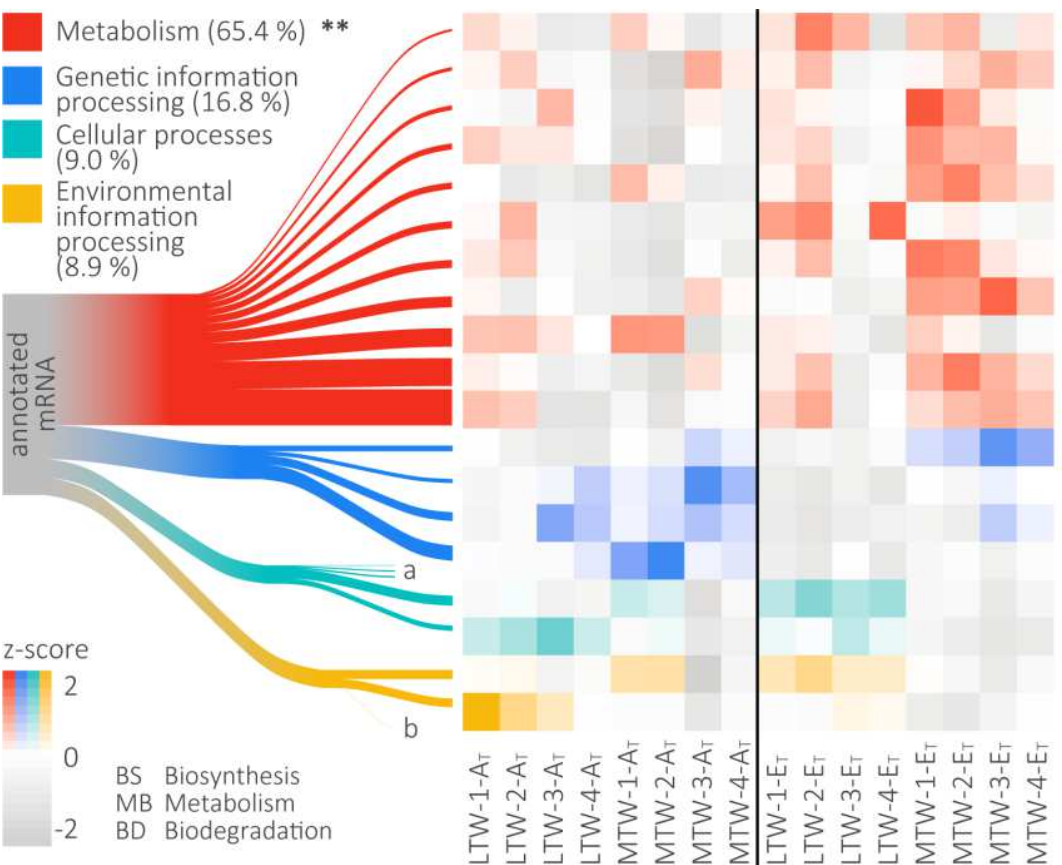

Glycan BS \& MB (1.2\%)

$\mathrm{MB}$ of terpenoids \& polyketides $(1.8 \%)$

BS of other secondary metabolites (2.3\%)

$\mathrm{MB}$ of other amino acids (3.0\%)

Nucleotide MB (3.2\%) +

Xenobiotics BD \& MB (3.6\%) +

Lipid MB (3.9\%) +

$\mathrm{MB}$ of cofactors \& vitamins $(5.4 \%)$

Energy MB (9.1\%)

Amino acid MB (13.8\%)

Carbohydrate MB (17.9\%) +

Replication \& repair $(2.8 \%)$

Transcription (2.0\%) *

Folding, sorting \& degradation $(4.4 \%) *$

Translation (7.6\%) *

Cellular community - prokaryotes (4.9\%)

Cell growth \& death ( $2.6 \%)$

Membrane transport ( $4.4 \%$ )

Signal transduction (4.5\%)

Transport \& catabolism $(0.8 \%)$

Cell motility $(0.6 \%$

Cellular community eukaryotes (0.2\%)

Signaling molecules \& interaction $(0.003 \%)$

Fig. 3. Functional annotation of mRNA reads. Sankey diagram showing the mean relative abundances of KEGG1 and KEGG2

categories over all samples. The heatmap depicts the relative abundances of all KEGG2 categories with mean relative

abundances $>1 \%$; samples are sorted by soil temperature $\left(\mathrm{A}_{\mathrm{T}}\right.$, ambient soil temperature; $\left.\mathrm{E}_{\mathrm{T}},+6{ }^{\circ} \mathrm{C}\right)$. The heatmap colour

code indicates the KEGG1 affiliation of the KEGG2 categories. Potential warming-induced differences in mRNA abundances Table 8).

\section{Community-wide shifts in gene expression of central metabolisms and cellular functions}

Since the gene expression patterns observed within the broad functional categories suggested

functional temperature-dependencies (Fig. 3) we screened all KEGG3 categories to identify which

specific metabolic pathways and functional complexes were the basis for these overall patterns.

One third of all functionally annotated mRNAs were affiliated with KEGG3 categories that exhibited 
in $\mathrm{E}_{\mathrm{T}}$ and encompassed central $\mathrm{C}$ metabolisms and metabolic pathways associated with amino acids and nucleotides, while five showed higher relative expression levels in $A_{T}$ and included protein biosynthesis related complexes and Oxidative phosphorylation (i.e. ATP formation) (Fig. 4a) . This change in the transcript abundances for the most central metabolisms in organismal function was observed for multiple taxa (Fig. 4b). In the long-term warmed soils, four phyla, eight classes, four orders, and four families, representing $33.9 \%, 38.1 \%, 11.1 \%$, and $1.8 \%$ of the LTW metatranscriptomes, respectively, displayed this pattern, confirming that this is a taxonomically broad type of warming response. This was supported by the analysis of the medium-term warmed soils, where seven phyla, 14 classes, seven orders, and eleven families, representing $87.2 \%, 65.0 \%$, $24.1 \%$, and $6.0 \%$ of the MTW metatranscriptomes, respectively, displayed the same expression pattern. It should be noted that the low percentages at family level is due to an average of only $22.1 \%$ of the annotated mRNA reads being assigned to a family-level taxon. Interestingly, the pattern occurred in taxa (Fig. 4b taxa in bold) regardless of whether these taxa became more active (higher relative abundance of mRNA and rRNA) or less active with warming (Fig. 2, Supplementary Fig. 3), indicating that the physiology of these community members had been altered in a similar way. Furthermore, our extensive screening revealed that on average this was true for nearly two thirds (63.7\%) of the taxa present in LTW and MTW (Fig. 4c, Supplementary Fig. 6). However, the percentage of taxa expressing the pattern varied between 43 and $95 \%$ depending on the KEGG3 category and the warming duration and was highest for the KEGG3 categories related to RNA, protein and energy metabolisms. 

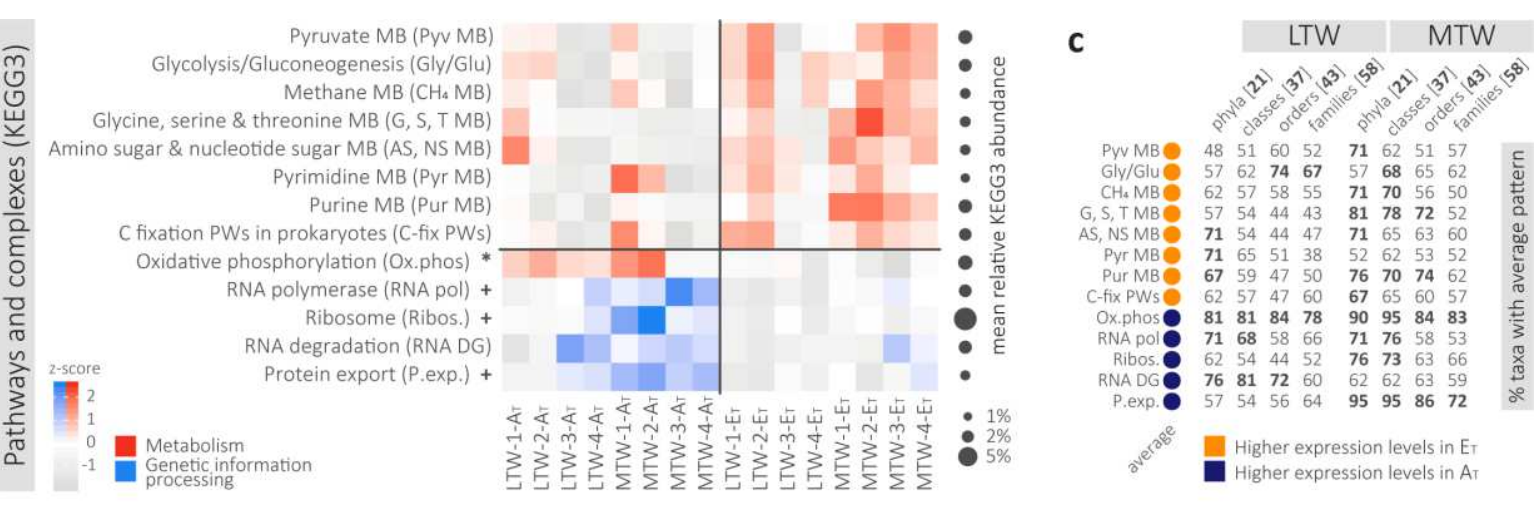

b

LTW
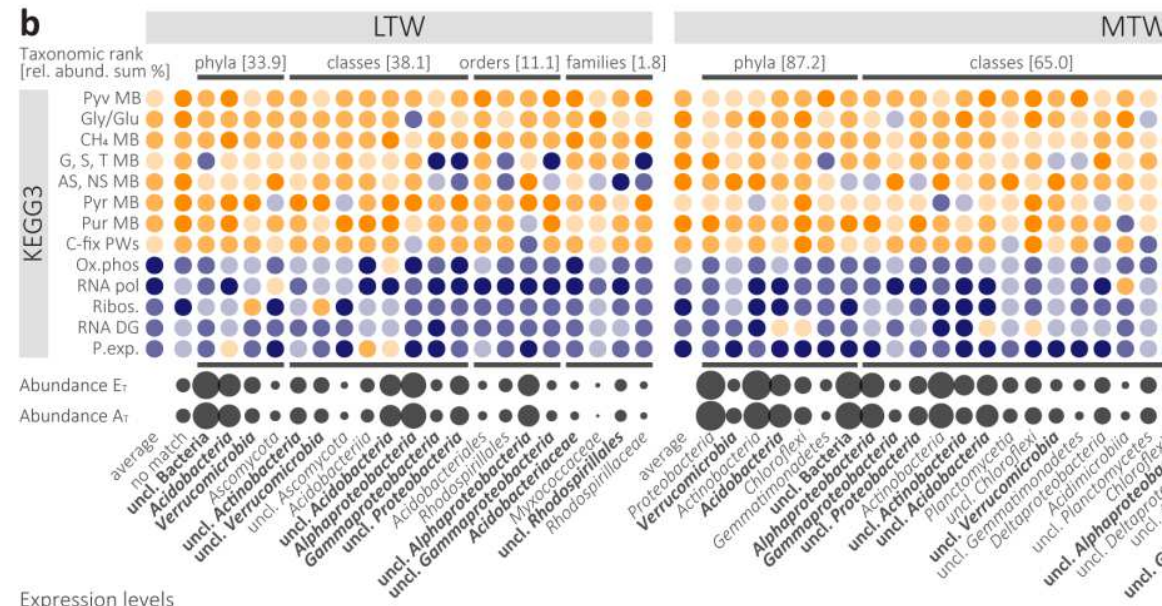

Expression levels

$E_{T}$ mean $>A_{-}$mean $\quad E_{T}$ mean $>A_{-}$mean $+3 / 4$ highest expression levels in $E_{1}$

$A_{T}$ mean $>E_{T}$ mean $A_{T}$ mean $>E_{T}$ mean $+3 / 4$ highest expression levels in $A_{T}$

Phyla [87.2]

MTW

orders [24.1]

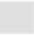

Fig. 4. Overall and taxon-specific warming-induced transcript abundance patterns. a Pathways and complexes with mean

223 relative abundances $\geq 1 \%$ that passed the differential abundance-pattern filter (13/16-filter, see Methods). Colour code

indicates the corresponding KEGG1 category. Differential gene expression analysis results are indicated next to the KEGG 3 categories $\left(\mathrm{n}=16, P_{\text {corr }}<0.05\left({ }^{*}\right), P_{\text {corr }}<0.1(+)\right.$; Supplementary Table 8). b Taxa reflecting the observed warming-induced abundance patterns presented in (a). All taxa with a mean relative abundance $\geq 1 \%$ in $L T W-A_{T}, L T W-E_{T}, M T W-A_{T}$, or MTW-ET and not more than two deviations from the overall pattern are depicted. Numbers in square brackets next to the taxonomic ranks are the sums of the relative abundances of the individual taxa represented below (grey circles). Colour code indicates the mean relative abundance of a KEGG3 category: orange, higher expression levels in $\mathrm{E}_{\mathrm{T}}$; blue, higher expression levels in $\mathrm{A}_{\mathrm{T}}$. Pale, dark, and intermediate colours indicate how strong and widely distributed a pattern was across LTW and MTW soils, respectively (see legend "Expression levels"). See (a) for KEGG3 abbreviations. c Table listing the percentage of all abundant taxa (i.e. taxa with $>1 \%$ o relative abundance) within a taxonomic rank that featured the overall warming-induced abundance pattern of a specific KEGG3 category (see Supplementary Fig. 6 for details on the taxa). Numbers in square brackets next to the taxonomic ranks give the total number of abundant taxa within the taxonomic rank. Bold numbers indicate pathways and complexes with warming-induced differential abundance patters observed in more than two thirds of all abundant taxa within a taxonomic rank. 

abundances of Pyrimidine, and Purine metabolism transcripts were also seen (Fig. 4). This indicated an upregulation of the production of major building-blocks of nucleic acids and proteins in the warmed soils, prompting us to investigate warming effects on bacterial growth (cell division), energy metabolism, and protein biosynthesis in detail. The eukaryotic fraction was excluded from the further analyses due to the large variations between LTW and MTW (Fig. 2).

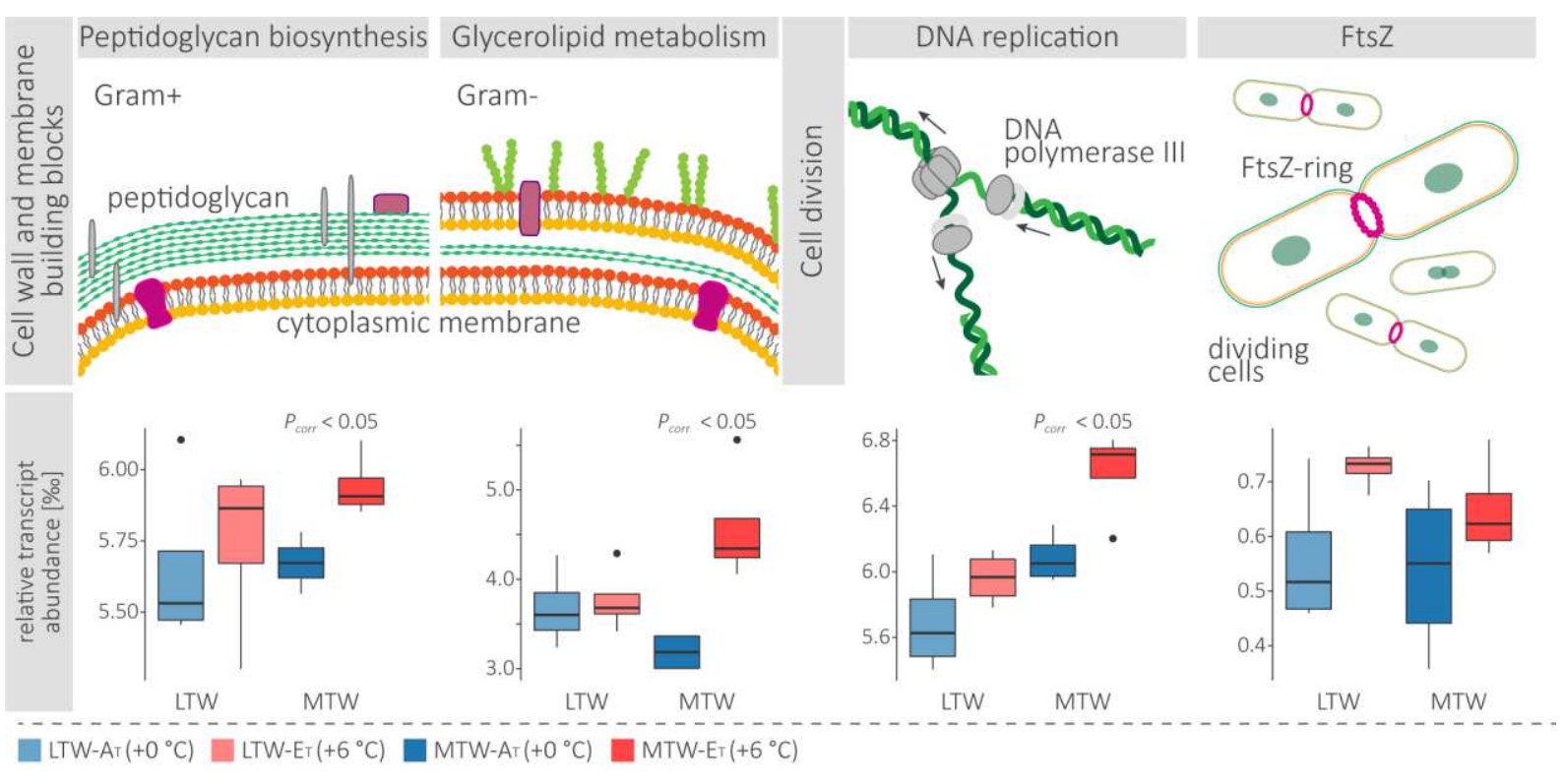

Fig. 5. Warming-induced abundance profiles of bacterial growth-related transcripts. Boxplots showing relative transcript abundances of the KEGG3 categories Peptidoglycan biosynthesis, Glycerolipid metabolism, and DNA replication, and FtsZ in the bacterial fractions of the LTW- $A_{T}, L T W-E_{T}, L T W-A_{T}$, and LTW- $E_{T}$ metatranscriptomes. The depicted KEGG3 categories are involved in the build-up of new cell walls and cell membranes and responsible for the duplication of genomic DNA, which precedes bacterial cell division; and FtsZ represents a key enzyme in bacterial cell division (see schematic drawings above the boxplots). P-values ( $\left.\mathrm{P}_{\text {corr }}\right)$ indicating significant differences are displayed above boxplot-pairs (Supplementary Table 9).

We observed trends of warming-induced transcriptional responses in a broad set of genes fundamental to bacterial cell replication (Fig. 5, Supplementary Table 9). In LTW, we observed 
increased relative transcript abundances for Peptidoglycan biosynthesis, DNA replication and FtsZ in $\mathrm{E}_{\mathrm{T}}$, albeit insignificant. However, in MTW- $\mathrm{E}_{\mathrm{T}}$, relative transcript abundances for Peptidoglycan biosynthesis, Glycerolipid metabolism, and DNA replication were significantly higher in MTW- $\mathrm{E}_{\mathrm{t}}$ than MTW-A $\left(\mathrm{t}\right.$-tests, $\mathrm{n}=8, P_{\text {corr }}<0.05$, Fig. 5), while the trend for FtsZ transcripts was similar but insignificant.

While the relative abundances of cell replication-related transcripts increased with warming, transcripts for energy conservation (Oxidative phosphorylation) showed the opposite trend. All four enzyme complexes of the membrane-bound prokaryotic electron transport chain and ATP synthase (producing ATP, the energy currency of cells) showed lower mean relative transcript abundances in $E_{T}$ (Fig. 5a). In contrast, transcripts of enzymes providing inorganic phosphate $\left(P_{i}\right)$ to the ATP synthase, especially polyphosphate kinase, showed higher mean relative abundances in $E_{T}$ than $A_{T}$.

Similar to Oxidative phosphorylation (i.e. ATP formation), transcripts for multiple complexes related to protein biosynthesis showed a trend towards lower relative abundances in $\mathrm{E}_{\mathrm{T}}$ (Fig. 6b). While insignificant after correcting for multiple testing $\left(P_{\text {corr }}>0.05\right.$, Supplementary Table 10$)$, such trends were also observed for RNA polymerase, ribosomal proteins, ribosomal RNA, the main molecular chaperones GroEL and DnaK (involved in protein folding), and the Sec-dependent pathway, the dominant protein export pathway present in the metatranscriptomes. Contrary to all other categories of the protein biosynthesis machinery, transcripts for RNA degrading complexes (RNA degradosomes) showed higher relative abundances in $E_{T}$ than $A_{T}$ (Fig. 6b).

We also investigated in detail the gene expression profiles for central $\mathrm{C}$ metabolisms. While the transcript abundances of KEGG3 categories Pyruvate metabolism, Glycolysis/Gluconeogenesis, Methane metabolism, and C fixation pathways in prokaryotes pointed towards higher expression levels in the warmed soils (Fig. 4), these differences were not significant. This suggests that while a shift in community metabolism is indicated, this might not be reflected consistently in all pathway steps and responsible bacteria that contribute to the above mentioned KEGG3 categories. 


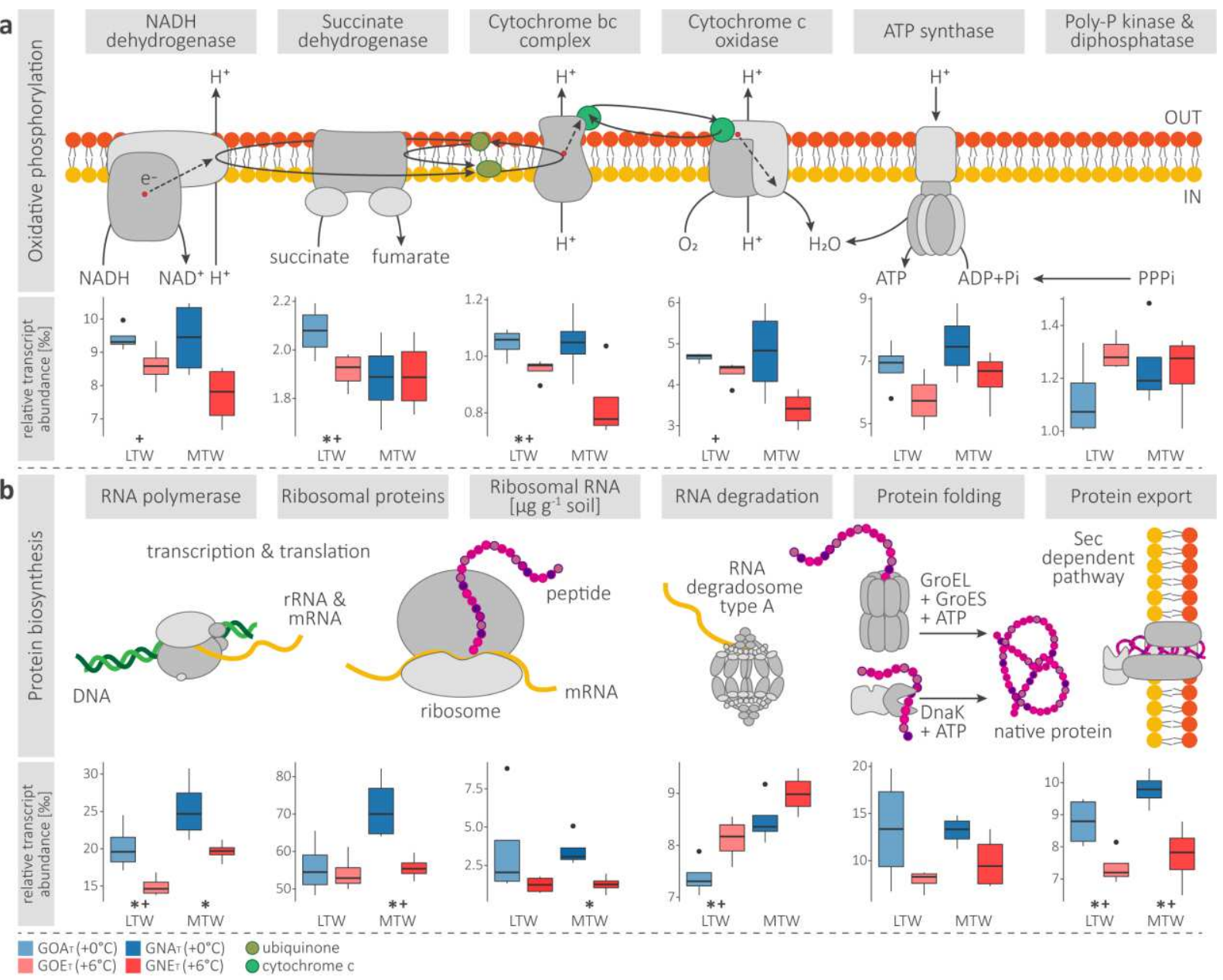

Fig. 6. Warming-induced abundance profiles of transcripts related to bacterial energy metabolism and protein

biosynthesis. Boxplots show the relative transcript abundances of enzymes and enzyme complexes involved in (a)

membrane-bound electron transport and ATP synthesis (oxidative phosphorylation) and (b) protein biosynthesis in the

LTW-A, LTW-ET, LTW-A

boxplots). Schematic representations of the enzymes and enzyme complexes are provided above each boxplot and are

based on the KEGG pathway drawings. Membrane-bound complexes are embedded in a lipid bilayer. Potential warming-

induced differences are indicated by $p$-values (uncorrected) $<0.05\left({ }^{*}\right)$ and corrected p-values $<0.1(+)(t$-tests, $n=8$,

Supplementary Table 10). GroEL and DnaK are part of the KEGG3 category RNA degradation, thus skewing its overall

warming-induced abundance pattern towards lower transcript abundances in $E_{T}$ than $A_{T}$ as seen in (Fig. 4a).

Finally, we examined, within all KEGG3 categories, the differential expression of single functions (KO).

Notably, the observed patterns, including those not significant at the KEGG3 level, were confirmed by multiple functions $(\mathrm{KO})$ that were differentially expressed and significant after multiple testing 
correction (Supplementary data S2). For functions related to protein biosynthesis and oxidative phosphorylation (ATP formation) the patterns were particularly strong and supportive of the above described trends. Less, although substantial, support was found for central carbon and amino acid metabolism patterns.

\section{Discussion}

Here we used metatranscriptomics to elucidate how soil microorganisms change their gene expression of central metabolic functions and cellular processes in response to warming. We showed that long-term $(>50 \mathrm{y})$, but not medium-term $(8 \mathrm{y})$, soil warming $\left(+6^{\circ} \mathrm{C}\right)$ resulted in a significant, albeit small, decrease of unique molecular functions encoded by soil Bacteria, Archaea, Fungi, and microbial Eukaryotes. However, irrespectively of the duration of warming, physiological responses to warming as shown by the transcriptional tuning of central metabolisms for $\mathrm{C}$, energy, protein biosynthesis, and growth, were consistent and common across many community members.

The apparent overexpression of genes involved in Pyruvate metabolism, Glycolysis/Gluconeogenesis, Methane metabolism, and C fixation pathways in prokaryotes indicate that long-term as well as medium-term warming affect the central cellular $\mathrm{C}$ metabolism of soil microorganisms. However, inconsistencies in the relative transcript abundances of subjacent KOs indicated that the perceived upregulation of these metabolisms does not involve all associated enzymes, reflecting either how metabolic pathway efficiency can be controlled by only a few rate-limiting steps ${ }^{24}$ or that the patterns created by the distinct up-regulation of pathways in some organisms are diluted by other organisms that share pathway steps but not the response pattern.

In contrast, the reduction in relative transcript abundances of oxidative phosphorylation complexes, RNA polymerases, protein processing enzymes, and ribosomal proteins were consistent across multiple enzyme subunits and pathway steps. A downregulation of the protein biosynthesis 
machinery is not immediately recognizable as a strategy to counteract substrate limitation or maximize growth rates. However, a reduction of ribosomes may be biochemically favourable under the warming conditions. Ribosomes are macromolecular complexes that are the sites of protein synthesis (translation). They consist of ribosomal proteins and rRNAs and are present in tens of thousands of copies per bacterial cell ${ }^{25}$. Thus, ribosomes can comprise over one third of the dry cell mass ${ }^{26}$ and rRNAs can account for $>90 \%$ of the total cellular RNA content ${ }^{27}$. Starving Escherichia coli and Salmonella spp. cells reduce their ribosomal content ${ }^{28,29}$, suggesting that a downregulation of the translation machinery, which accounts for up to $40 \%$ of total cellular proteins $s^{30}$, is metabolically favorable in nutrient-limited ecosystems ${ }^{31}$. Furthermore, carefully tuning concentrations of abundant proteins such as ribosomal proteins can also free resources for accelerating other reactions ${ }^{30}$. We not only demonstrated lower relative transcript abundances of ribosomal proteins and protein biosynthesis-related enzymes in the warmed soils, but our results also showed lower microbial biomass per gram of soil dry weight, and a decrease in RNA per gram dry soil that correlated significantly with the biomass. A significant decrease in microbial biomass per gram of soil dry weight in the warmed soils has been observed previously at the same site ${ }^{16}$. Furthermore, the transcriptional patterns for protein biosynthesis were consistent for many community members, regardless of their relative abundances of total mRNAs and rRNAs in the long- and medium-term warmed soils and the controls. Thus, a downregulation of protein biosynthesis-related enzymes and a reduction of ribosomes might be a common response of microorganisms, especially bacteria, to ecosystem warming and partly responsible for the lower microbial biomass in the warmed soils. It seems contradictory that we observed, at the same time, higher relative transcript abundances for enzymes related to cell division and biosynthesis of amino acid, the building-blocks of proteins. However, a reduced ribosomal content is not necessarily linked to lower protein synthesis rates or lower growth rates. It is long known that increased temperatures accelerate mRNA synthesis and the protein synthesis rate per ribosome (peptide chain elongation rate) in the model organism Escherichia coli ${ }^{32}$. Conversely, it was indicated more recently that lower growth rates induced by slower reaction rates 
are associated with an increased content of ribosomal proteins ${ }^{30,33}$. Likewise, an acceleration of

348 reaction rates in the membrane-bound electron transport chain and ATP synthesis (Oxidative

349 phosphorylation) despite lower expression might also have been driven by temperature and might be indicated by the higher relative transcript abundances of enzymes (i.e. polyphosphate kinase) providing $P_{i}$ to the ATP synthase in the warmed soils. Since the translation machinery accounts for up to $40 \%$ of total cellular protein $\mathrm{s}^{30}$ and protein biosynthesis is the most costly type of macromolecular synthesis ${ }^{34-36}$ the reduced number of ribosomes would furthermore allow conservation and reallocation of energy (ATP) from the generation of ribosomal proteins to the synthesis of metabolic proteins.

Accelerated reaction rates due to increased temperatures presumably allowed microorganisms to reduce their ribosomal content and their machinery for oxidative phosphorylation, liberating energy and matter for substrate acquisition and growth. Through this transition, soil warming may have led to metabolically more active microbial cells driven by temperature and a different partitioning of cellular resources. Previous observations of higher biomass-specific growth, respiration, organic $C$ uptake, and turnover rates in the same long-term warmed soils ${ }^{16}$ corroborates this interpretation.

These results are clearly not consistent with the suggested thermal acclimation of soil microorganisms used to explain a return to pre-warming SOM degradation rates after a few years of warming $^{11,12}$ (and references therein). Rather, the apparent thermal acclimation in soil respiration rates might be driven by a reduction in microbial biomass caused by reduced carbon and nutrient concentrations, as previously suggested ${ }^{16}$. However, our results point at a different type of microbial acclimation to warming where the physiological adjustments allow the microbial community members to maintain a high activity even after decades of warming, despite more limiting conditions.

The microbial functional responses were more pronounced and widespread in the microbial

371 communities in the medium-term warmed soils than in the long-term warmed soils reflecting the 
recently reported systemic overreaction to years versus decades of warming in the same soils ${ }^{15}$. The authors proposed that an initial acceleration of biotic activity due to warming led to rapid decreases in $\mathrm{C}, \mathrm{N}$, and $\mathrm{P}$ pools within the first years after the onset of warming, followed by a decrease of microbial and fungal biomass and system stabilisation within decades. Our taxonomic analyses of the mRNA transcript pools extended our insight into this proposed soil warming chronology; long-term, but not medium-term, soil warming resulted in differential abundances. Relative mRNA transcript abundances of several bacterial taxa, fungi, protists, and viruses were affected by long-term warming. This indicated shifts in trophic interactions (e.g. from top-down to bottom-up control of the prokaryotic community) and possibly a reduced importance of fungi, with repercussion on organic matter decomposition. Reduced Fungi:Bacteria ratios have been described previously in long- and short-term warming experiments of forest and grassland soils ${ }^{8,37}$ and Fungi appear to be more abundant and active at lower temperatures ${ }^{4,38}$ and in soils with low $\mathrm{pH}^{39,40}$. Besides the higher temperatures and slightly higher $\mathrm{pH}$ in the warmed ForHot grassland soils, the lower concentrations of various soil $\mathrm{C}, \mathrm{N}$ and $\mathrm{P}$ compounds and decreased soil aggregate sizes ${ }^{19}$ may explain the lower relative abundances and diversity of fungal mRNAs. The few and non-significant taxonomic differences between ambient and medium-term warmed soils suggest that while the soil microorganisms respond functionally, there is little effect on microbial community structure. However, we cannot exclude that the apparent lack of taxonomic response after eight years resulted from the high variation between the biological replicates. Our results somewhat contrasts previous studies on the ForHot grassland sites that reported generally little taxonomic shifts with warming ${ }^{16,20}$. However, these studies applied DNA-based methods (i.e., 16S gene and ITS amplicon sequencing) targeting the potential microbial community, including active cells, dormant cells, and relic DNA. These methods are also prone to biases from PCR and primers. In contrast, metatranscriptomics is PCR-free and deploys random hexamer-primers targeting prokaryotic, eukaryotic, and viral transcripts in parallel, thus allowing a comprehensive analysis of the active soil microbiome. Furthermore, our analyses did demonstrate a stronger effect of warming on microbial functions 
398 (KOs) than on taxonomy. This functional response was similar in medium-term and long-term

399 warmed soils. Thus, our study provides evidence for a decoupling of microbial community structure 400 and functions, as recently suggested from several ecosystems including soil ${ }^{41-44}$.

401 How warming affects the microbial control of the global C cycle is a key question to better

402 understand the soil-climate feedbacks, an answer to which is urgently needed ${ }^{4,45}$. Here, for the first

403 time in a comprehensive study of the transcriptional response of microorganisms across all domains

404 of life, we show that downregulation of energy metabolism and protein biosynthesis is central in the

405 microbial soil warming response that allow microorganisms to maintain high metabolic activities and

406 cell division rates even after decades of warming. We suggest that accelerated biochemical reaction

407 rates due to higher temperatures, have a positive feedback on central metabolisms via increased

408 relative transcript abundances, if not constraint by reduced substrate concentrations, further

409 accelerating microbial decomposition of $\mathrm{SOM}$ and the subsequent release of $\mathrm{CO}_{2}$ to the atmosphere. 


\section{Grassland sites and soil sampling}

Soil samples were collected from a natural grassland near Hveragerđi $\left(64^{\circ} 0^{\prime} \mathrm{N}, 21^{\circ} 11^{\prime} \mathrm{W}\right)$, Iceland, in July 2016. The grassland is part of the ForHot experiment ${ }^{14}$ and features two sites, each consisting of replicated soil temperature gradients that were formed by natural geothermal activity. One site has experienced warming for $>50 \mathrm{y}$, possibly since before $1708^{14}$. Geothermal activities may have varied over time, but warming has been stable in the area since at least 1963, and the warming intensities of the temperature gradients have not varied since detailed measurements began ${ }^{14-16}$. The second site recently developed similar temperature gradients after an earthquake in 2008 . We collected 16 samples that were later analysed in detail; i.e. four samples of long-term warmed soils (LTW) exposed to elevated temperatures $\left(+6^{\circ} \mathrm{C}\right.$ above ambient, $\left.\mathrm{LTW}-\mathrm{E}_{\mathrm{T}}\right)$ and four corresponding controls at ambient temperatures (LTW-AT) as well as four samples of medium-term (8 y) warmed soils (MTW) exposed to $+6{ }^{\circ} \mathrm{C}$ above ambient temperatures $\left(M T W-E_{T}\right.$ ) and four corresponding controls (MTW- $\left.A_{T}\right)$. The grassland soils are classified as Silandic Andosols, and both sites are dominated by Agrostis capillaris with varying undergrowth and vascular pant and moss cover ${ }^{14}$. We took soil samples $(0-10$ $\mathrm{cm}$ depth) from ambient and $+6{ }^{\circ} \mathrm{C}$ plots of four replicate blocks at one time point. Samples were immediately frozen in liquid $\mathrm{N}_{2}$ for subsequent nucleic acid extraction and metatranscriptomics after sieving to $2 \mathrm{~mm}$.

\section{Physicochemical soil properties}

Fresh aliquots of each soil sample were extracted with either $1 \mathrm{M} \mathrm{KCl}$ or $0.5 \mathrm{M} \mathrm{NaHCO}_{3}$ solution for $30 \mathrm{~min}$ at room temperature or fumigated with chloroform for $48 \mathrm{~h}$ and subsequently extracted using the same extractants. Various soil C and $\mathrm{N}$ compounds and soil P compounds (Supplementary Table 1) were quantified in the $\mathrm{KCl}$ and $\mathrm{NaHCO}_{3}$ extracts, respectively, using standard procedures ${ }^{46}$. $\mathrm{C}_{\mathrm{MO}}, \mathrm{N}_{\mathrm{MO}}$, and $\mathrm{P}_{\mathrm{MO}}$ were calculated as the differences between DOC, TDN, and TDP contents in the fumigated and non-fumigated extracts. Total $\mathrm{C}$ and $\mathrm{N}$ contents and stable isotope ratios were 
analysed in dried soil aliquots using an elemental analyser coupled to an isotope ratio mass spectrometer (EA-IRMS; EA1110 coupled via a ConFlo III interface to a DeltaPLUS IRMS, Thermo Fisher Scientific). Soil pH was determined from sieved soil samples in $0.05 \mathrm{M} \mathrm{CaCl}_{2}$ solution and gravimetric water content was determined.

\section{Nucleic acid extractions and sequencing}

We extracted total nucleic acids from 16 sieved soil samples (Supplementary Table 7), i.e. four replicates from each of the four sampled soil groups ( $\left.L T W-A_{T}, L T W-E_{T}, M T W-A_{T}, M T W-E_{T}\right)$, representing two warming intensities $\left(\mathrm{A}_{\mathrm{T}},+0^{\circ} \mathrm{C}\right.$, and $\left.\mathrm{E}_{\mathrm{T}},+6^{\circ} \mathrm{C}\right)$ of $>50 \mathrm{y}$ warmed soils $(\mathrm{LTW})$ and $8 \mathrm{y}$ warmed soils (MTW). These samples were selected because $6{ }^{\circ} \mathrm{C}$ above ambient is within the predicted and most severe range of (soil) warming over the next 60-100 $y^{47,48}$. Each sample was extracted in triplicate, as previously described ${ }^{49}$. Briefly, a phosphate buffer, a detergent solution containing $C T A B$, and TE saturated phenol $(\mathrm{pH}$ 8) were added to $0.3 \mathrm{~g}$ of soil in a lysis matrix $\mathrm{E}$ tube (MP Biomedicals) containing silica beads and shaken vigorously for $30 \mathrm{~s}\left(6.5 \mathrm{~m} \mathrm{~s}^{-1}\right)$ in a FastPrep machine (MP Biomedicals). After centrifugation, the aqueous supernatant was retained. This procedure was repeated two more times using fresh buffer, detergent, and phenol. The three supernatants were then pooled, followed by phenol:chloroform:isoamylalcohol (25:24:1) and chloroform:isoamylalcohol (24:1) extraction and precipitation of the nucleic acids using PEG 8000. The nucleic acids were treated with DNase (RQ1, Promega) before the metatranscriptomes were generated. RNA quantity and quality were assessed by automated agarose gel electrophoresis

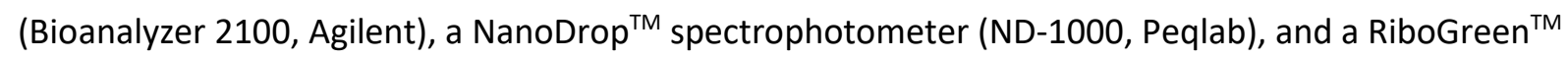
assay kit (Thermo Fisher Scientific). Total RNA was amplified using the MessageAmp II-Bacteria Kit (Ambion Life Technologies) with an input of 100 ng RNA, following the kit protocol, except the RNA was linearly amplified for $14 \mathrm{~h}$. The three technical replicates of each of the 16 samples were pooled prior to the amplification. Overlapping paired-end 125-bp reads were sequenced using the HighSeq2500 sequencer (Illumina) at the Vienna Biocenter Core Facilities, Vienna, Austria. 
462

463

464

465

466

467

468

469

470

471

472

\section{3}

474

475

We performed the following pre-processing steps and the majority of the subsequent analyses using the Life Science Compute Cluster (LiSC) run by CUBE (Division of Computational Systems Biology), Department of Microbiology and Ecosystem Science, University of Vienna, Austria. PEAR v.0.9.10 (Paired-End reAd mergeR, default settings) was used to merge the raw paired-end reads ${ }^{50}$. We subsequently used SortMeRNA v.2.1 to filter out non-rRNA reads from the total RNA reads ${ }^{51}$. The non-rRNA reads were quality filtered (-min_len 180 -max_len 240 -min_qual_mean 30 -ns_max_n 5 -trim_tail_right 15 -trim_tail_left 15) using prinseq-lite v.9.20.4 ${ }^{52}$. A second filtering step was performed to obtain mRNA reads and reduce the size of the dataset for later analyses. All non-rRNA reads that aligned to any sequence in the NCBI $n r^{53}$ database (as of September 2018) using a nonconservative DIAMOND blastx ${ }^{54}$ search (v.0.9.18, -k 1 -e 0.001) were kept. See Supplementary Table 7 for more details.

\section{Functional and taxonomic annotation}

We functionally annotated the total mRNA reads (Supplementary Table 7) by aligning them to the KEGG $^{23}$ database (as of April 2019) using a DIAMOND blastx ${ }^{54}$ search (v.0.9.18, -k 1 --min-score 52.5). A prior analysis indicated that at a bit score of 52.5 equalled an e-value of $\leq 0.0001$ (DIAMOND blastx of the same dataset against the KEGG database as of February 2019). We, therefore, used a bit score of 52.5 rather than an e-value of 0.0001 for all following analyses to obtain a better comparability between different database searches, independent of increasing database sizes. We performed a DIAMOND blastx ${ }^{54}$ search (v.0.9.25, -k 25 --min-score 52.5) against the NCBI nr ${ }^{53}$ database (as of October 2019) to obtain taxonomic information for the mRNA reads. We used the program blast2lca, the standalone implementation of the MEGAN (v.6.11.1) LCA (lowest common ancestor)-assignment algorithm, to obtain one taxonomic assignment for each read based on the $25 \mathrm{nr}$ database hits ${ }^{55}$. The following parameters were used to obtain the LCAs: -ms 50 -me 0.01 -top 5 -mid 0.0 (mapping file: prot_acc2tax-Jul2019X1.abin). Subsamples of 200,000 rRNA reads were taxonomically annotated as previously described ${ }^{43}$. 


\section{Data analysis}

488 We used $\mathrm{R}^{56}$ to analyse the data, perform statistical tests, and graphically display the results (Rstudio

489

490

491

492

493

494

495 version 1.1.456) including the R packages ggalluvial version 0.10 .0 (https://CRAN.Rproject.org/package=ggalluvial), ggplot2 version 3.2.0 (https://CRAN.R-project.org/package=ggplot2), vegan version 2.5-6 (https://CRAN.R-project.org/package=vegan), and edgeR version 3.32.0 (https://bioconductor.org/packages/edgeR). Further details on the R packages can be found in the respective sections. Functionally annotated and taxonomically classified mRNA datasets were merged. Functionally annotated mRNA reads that lacked a taxonomic classification were tagged with "no match". The resulting data were normalised and filtered.

Filtering and normalisation. First, we normalised the data to the library size and transferred them to permille (\%). Second, only functions (KOs, KEGG database entries) present in all four replicates of at least one group ( $L T W-A_{T}, L T W-E_{T}, M T W-A_{T}$, or MTW-E $E_{T}$ ) were kept (removing $0.6 \%$ of the data). Third, low abundant functions were removed by filtering out KOs with a total relative abundance (sum of all 16 datasets) below $<0.1 \%$ (removing $4.6 \%$ of the data remaining after the previous step), which equalled a mean relative abundance of a specific function per sample of $<0.00625 \%$ (mean relative abundance $[\% \circ]=0.1 \% / 16$ ). A second dataset containing all taxonomically classified mRNA reads (without functional annotation) was likewise normalised and transferred. The taxonomy-only dataset was filtered by removing all families (taxonomic strings from domain to family) that were not present in all replicates of at least one group (LTW-AT, LTW-ET, MTW- $A_{T}$, or MTW- $E_{T}$ ) (removing $0.07 \%$ of the data); no second filter was applied. Viral reads were summarised prior to filtering depending on the subsequent analyses.

Trend charts. Trend charts (e.g. Figure 1C) were created by calculating the means of $A_{T}$ and $E_{T}$ replicates, respectively (LTW and MTW separate and combined) and comparing them to the mean over all samples ( $A_{T}$ and $E_{T}$ combined, LTW and MTW separate and combined) which was set to 1 . The shapes of the individual wedges reflect the magnitude of the differences. Wedges are coloured alternating (brown and grey) to ease discriminability. Dark brown and dark grey indicate values $>1$ 
513 (i.e. above the over-all mean) while light brown and light grey indicate values $<1$ (i.e. below the over-

514 all mean).

515 Heatmaps. Heatmaps were generated using the geom_tile function of the ggplot2 R package. Before plotting, normalised data were transformed by z-scoring, either over all 16 samples or separately for TLW and MTW. Two explorative filters were subsequently applied for selecting patterns of interest. Abundance patterns of taxa present in LTW and MTW, respectively, were subject to a stringent filter (referred to as 4/4-filter); only taxa with higher or lower relative abundances in all four replicates of one group relative to their counterparts passed the filter threshold (e.g. a taxon passes the filter if the four highest values are found in LTW-A and the four lowest in LTW-ET). A less stringent filter (referred to as 13/16-filter) was applied when the relative abundances of KEGG functions between $A_{T}$ and $E_{T}$ across all samples were compared (e.g. Figure 4). Patterns were retained only if: $i$ ) at least six samples of one temperature group $\left(A_{T}\right.$ or $E_{T}$ ) were higher than the third highest sample of the other temperature group $\left(E_{T}\right.$ or $\left.A_{T}\right)$ and at least seven samples of one temperature group $\left(E_{T}\right.$ or $\left.A_{T}\right)$ were lower than the third lowest sample of the other temperature group $\left(A_{T}\right.$ or $\left.E_{T}\right)$ or ii) at least seven samples of one temperature group $\left(A_{T}\right.$ or $E_{T}$ ) were higher than the third highest sample of the other temperature group ( $E_{T}$ or $A_{T}$ ) and at least six samples of one temperature group ( $E_{T}$ or $A_{T}$ ) were lower than the third lowest sample of the other temperature group $\left(A_{T}\right.$ or $\left.E_{T}\right)$. Therefore, the critical threshold to pass the $13 / 16$-filter lies at $80 \%$ consensus with the most stringent warming-associated distribution (i.e. the eight highest relative transcript abundances are found in one temperature group and the eight lowest in the opposite temperature group).

Alluvial plots. Alluvial plots (Sankey diagrams) were created using the R package ggalluvial. Individual Sankey diagrams were manually merged if more than two levels were shown.

Boxplots. Boxplots were generated using geom_boxplot (R package ggplot2).

Non-metric multidimensional scaling (NMDS). NMDS was used to obtain ordination plots depicting

537 (dis)similarities between the microbial functions and microbial community structures of the samples.

538 We used the metaMDS function implemented in the R package vegan, two dimensions, and a 
maximum of 10,000 random starts in search of a stable solution. The sequencing data were normalised and filtered as described above prior to the NMDS analyses. GUSTA ME (GUide to STatistical Analysis in Microbial Ecology ${ }^{57}$ was consulted for selecting the appropriate dissimilarity index (i.e. "canberra").

Statistics and post hoc analyses. The basic R function t.test was used to perform two-sided Student's $t$-Tests to identify significant differences between $A_{T}$ and $E_{T}$ (of LTW and MTW, respectively, or across LTW and MTW). Obtained p-values were corrected for multiple testing (Benjamini-Hochberg procedure, basic $\mathrm{R}$ function p.adjust). Corrected $\mathrm{p}$-values $\left(\mathrm{P}_{\text {corr }}, \mathrm{q}\right.$-values $)<0.05$ were considered to indicate significant differences. We deliberately chose a parametric test (decreasing the chance of making a type II error) combined with multiple-testing adjustment and considered corrected p-values $<0.1$ to indicate a temperature-dependent trend, reflecting the explorative nature ${ }^{58}$ of our study. The basic $\mathrm{R}$ function cor.test was used to identify associations between microbial biomass and RNA content, DNA content, and various $\mathrm{C}, \mathrm{N}$, and $\mathrm{P}$ concentrations by applying Spearman's rank correlation (two-sided). The ggplot function geom_smooth was used to indicate correlations (method $=\mathrm{Im}$ ). Taxonomic (family) and functional (KO) richness was estimated from raw read counts (families and KOs not present in all four replicates of at least one group (LTW-AT, LTW- $E_{T}, M T W-A_{T}$, or MTW-ET) were considered as noise and excluded) using the vegan function rarefy. Log2-fold changes in transcript abundance between temperature group $\left(\mathrm{GOA}_{T}, \mathrm{GOE}_{T}, \mathrm{GNA}_{T}\right.$, and $\left.\mathrm{GNE}_{\mathrm{T}}\right)$ means were calculated in $\mathrm{R}: \log 2\left(\right.$ mean $\mathrm{E}_{\mathrm{T}} /$ mean $\left.\mathrm{A}_{\mathrm{T}}\right)$. Permutational multivariate analysis of variance (PERMANOVA, vegan function adonis) was used to identify the effect of warming $\left(A_{T}\right.$ and $\left.E_{T}\right)$ and warming duration (LTW and MTW), respectively, on the distribution of samples (see NMDS plots) by physicochemical soil properties (including microbial biomass measures, DNA, and RNA concentrations) or gene expression. In all cases, 10,000 permutations were calculated, and the dissimilarity indices were the same as those used in the NMDS analyses (see above). Differential gene expression analyses on taxonomic and functionally annotated datasets were performed using edgeR (function glmQLFTest). Raw read counts of families or functions (KOs, KEGG database entries) 
565 present in all four replicates of at least one group ( $L T W-A_{T}, L T W-E_{T}, M T W-A_{T}$, or MTW- $\left.E_{T}\right)$ were used

566 as input data. Low abundant taxa (kingdoms, phyla, classes orders, and families $<1 \%$ ), low abundant

567 KEGG categories (KEGG 1, KEGG 2, and KEGG $3<1 \%$ ), and low abundant functions ( $K O<0.5 \%$ ) were

568 excluded from the analyses and the default trimmed mean of M-values normalization (TMM) method

569 was used to normalize the data.

570 Adobe Illustrator (CC 23.0.2.) was used for final figure editing.

571 


\section{References}

573 1. Allen, M. R. et al. Framing and Context. in Global Warming of $1.5^{\circ} \mathrm{C}$. An IPCC Special Report on

574 the impacts of global warming of $1.5^{\circ} \mathrm{C}$ above pre-industrial levels and related global greenhouse

575

576 gas emission pathways, in the context of strengthening the global response to the threat of climate change, sustainable development, and efforts to eradicate poverty. (IPCC, 2018).

2. Ciais, P. et al. Carbon and Other Biogeochemical Cycles. in Climate Change 2013: The Physical Science Basis. Contribution of Working Group I to the Fifth Assessment Report of the Intergovernmental Panel on Climate Change. (eds Stocker et al.). (IPCC AR5 WG1, 2013).

3. Canadell, J. G. et al. Contributions to accelerating atmospheric $\mathrm{CO}_{2}$ growth from economic activity, carbon intensity, and efficiency of natural sinks. Proc. Natl. Acad. Sci. U. S. A. 104, 18866-18870 (2007).

4. Jansson, J. K. \& Hofmockel, K. S. Soil microbiomes and climate change. Nat. Rev. Microbiol. 18, 35-46 (2020).

5. Kallenbach, C. M., Frey, S. D. \& Grandy, A. S. Direct evidence for microbial-derived soil organic matter formation and its ecophysiological controls. Nat. Commun. 7, (2016).

6. Liang, C., Amelung, W., Lehmann, J. \& Kästner, M. Quantitative assessment of microbial necromass contribution to soil organic matter. Glob. Chang. Biol. 25, 3578-3590 (2019).

7. Wang, K. et al. Modeling Global Soil Carbon and Soil Microbial Carbon by Integrating Microbial Processes into the Ecosystem Process Model TRIPLEX-GHG. J. Adv. Model. Earth Syst. 9, 23682384 (2017).

8. Melillo, J. M. et al. Long-term pattern and magnitude of soil carbon feedback to the climate system in a warming world. Science 358, 101-105 (2017).

9. Romero-Olivares, A. L., Allison, S. D. \& Treseder, K. K. Soil microbes and their response to experimental warming over time: A meta-analysis of field studies. Soil Biol. Biochem. 107, 32-40 (2017). 
10. Knorr, W., Prentice, I. C., House, J. I. \& Holland, E. A. Long-term sensitivity of soil carbon turnover to warming. Nature 433, 298-301 (2005).

11. Carey, J. C. et al. Temperature response of soil respiration largely unaltered with experimental warming. Proc. Natl. Acad. Sci. U. S. A. 113, 13797-13802 (2016).

12. Bradford, M. A. Thermal adaptation of decomposer communities in warming soils. Front. Microbiol. 4, 333 (2013).

13. Kirschbaum, M. U. F. Soil respiration under prolonged soil warming: Are rate reductions caused by acclimation or substrate loss? Glob. Chang. Biol. 10, 1870-1877 (2004).

14. Sigurdsson, B. D. et al. Geothermal ecosystems as natural climate change experiments: The ForHot research site in Iceland as a case study. Icelandic Agric. Sci. 29, 53-71 (2016).

15. Walker, T. W. N. et al. A systemic overreaction to years versus decades of warming in a subarctic grassland ecosystem. Nat. Ecol. Evol. 4, 101-108 (2020).

16. Walker, T. W. N. et al. Microbial temperature sensitivity and biomass change explain soil carbon loss with warming. Nat. Clim. Change 8, 885-889 (2018).

17. Marañón-Jiménez, S. et al. Geothermally warmed soils reveal persistent increases in the respiratory costs of soil microbes contributing to substantial C losses. Biogeochemistry 138, 245260 (2018).

18. Marañón-Jiménez, S. et al. Coupled carbon and nitrogen losses in response to seven years of chronic warming in subarctic soils. Soil Biol. Biochem. 134, 152-161 (2019).

19. Poeplau, C., Kätterer, T., Leblans, N. I. W. \& Sigurdsson, B. D. Sensitivity of soil carbon fractions and their specific stabilization mechanisms to extreme soil warming in a subarctic grassland. Glob. Chang. Biol. 23, 1316-1327 (2017).

20. Radujković, D. et al. Prolonged exposure does not increase soil microbial community compositional response to warming along geothermal gradients. FEMS Microbiol. Ecol. 94, 174 (2018). 
21. Urich, T. et al. Simultaneous assessment of soil microbial community structure and function through analysis of the meta-transcriptome. PLoS One 3, e2527 (2008).

22. Bushmanova, E., Antipov, D., Lapidus, A. \& Prjibelski, A. D. RnaSPAdes: A de novo transcriptome assembler and its application to RNA-Seq data. Gigascience 8, (2019).

23. Kanehisa, M. \& Goto, S. KEGG: Kyoto Encyclopedia of Genes and Genomes. Nucleic Acids Res. 28, $27-30$ (2000).

24. Rognstad, R. Rate-limiting steps in metabolic pathways. J. Biol. Chem. 254, 1875-1878 (1979).

25. Bremer, H. \& Dennis, P. P. Modulation of Chemical Composition and Other Parameters of the Cell by Growth Rate. in Escherichia coli and Salmonella typhimurium: Cellular and Molecular Biology. (eds. Neidhardt, F. C. et al.). (ASM Press, Washington, 1996).

26. Milo, R. \& Phillips, R. Cell biology by the numbers (Garland Science, New York City, 2015).

27. Blazewicz, S. J., Barnard, R. L., Daly, R. A. \& Firestone, M. K. Evaluating rRNA as an indicator of microbial activity in environmental communities: Limitations and uses. ISME J. 7, 2061-2068 (2013).

28. Davis, B. D., Luger, S. M. \& Tai, P. C. Role of ribosome degradation in the death of starved Escherichia coli cells. J. Bacteriol. 166, 439-445 (1986).

29. Hsu, D., Shih, L. M. \& Zee, Y. C. Degradation of rRNA in Salmonella strains: A novel mechanism to regulate the concentrations of rRNA and ribosomes. J Bacteriol. 176, 4761-4765 (1994).

30. Bosdriesz, E., Molenaar, D., Teusink, B. \& Bruggeman, F. J. How fast-growing bacteria robustly tune their ribosome concentration to approximate growth-rate maximization. FEBS J. 282, 20292044 (2015).

31. Klappenbach, J. A., Dunbar, J. M. \& Schmidt, T. M. rRNA operon copy number reflects ecological strategies of bacteria. Appl. Environ. Microbiol. 66, 1328-1333 (2000).

32. Ryals, J., Little, R. \& Bremer, H. Temperature dependence of RNA synthesis parameters in Escherichia coli. J. Bacteriol. 151, 879-887 (1982). 
33. Scott, M., Gunderson, C. W., Mateescu, E. M., Zhang, Z. \& Hwa, T. Interdependence of cell growth and gene expression: Origins and consequences. Science 330, 1099-1102 (2010).

34. Stouthamer, A. H. A theoretical study on the amount of ATP required for synthesis of microbial cell material. Antonie Van Leeuwenhoek 39, 545-565 (1973).

35. Russell, J. B. The energy spilling reactions of bacteria and other organisms. J. Mol. Microbiol.

$$
\text { Biotechnol. 13, 1-11 (2007). }
$$

36. Buttgereit, F. \& Brand, M. D. A hierarchy of ATP-consuming processes in mammalian cells.

$$
\text { Biochem. J. 312, 163-167 (1995). }
$$

37. Zhang, B. et al. Responses of soil microbial communities to experimental warming in alpine grasslands on the Qinghai-Tibet Plateau. PLoS One 9, e103859 (2014).

38. Pietikäinen, J., Pettersson, M. \& Bååth, E. Comparison of temperature effects on soil respiration and bacterial and fungal growth rates. FEMS Microbiol. Ecol. 52, 49-58 (2005).

39. Weyman-Kaczmarkowa, W. \& Pędziwilk, Z. The development of fungi as affected by pH and type of soil in relation to the occurrence of bacteria and soil fungistatic activity. Microbiol. Res. 155, 107-112 (2000).

40. Högberg, M. N., Högberg, P. \& Myrold, D. D. Is microbial community composition in boreal forest soils determined by pH, C-to-N ratio, the trees, or all three? Oecologia 150, 590-601 (2007).

41. Frossard, A., Gerull, L., Mutz, M. \& Gessner, M. O. Disconnect of microbial structure and function: Enzyme activities and bacterial communities in nascent stream corridors. ISME J. 6, 680-691 (2012).

42. Purahong, W. et al. Uncoupling of microbial community structure and function in decomposing litter across beech forest ecosystems in Central Europe. Sci. Rep. 4, 1-7 (2014).

43. Söllinger, A. et al. Holistic Assessment of Rumen Microbiome Dynamics through Quantitative Metatranscriptomics Reveals Multifunctional Redundancy during Key Steps of Anaerobic Feed Degradation. mSystems 3, e00038-18 (2018).

44. Min, K. et al. Temperature sensitivity of biomass-specific microbial exo-enzyme activities and CO 

2 efflux is resistant to change across short- and long-term timescales. Glob. Chang. Biol. 25, 1793-1807 (2019).

45. Bardgett, R. D., Freeman, C. \& Ostle, N. J. Microbial contributions to climate change through carbon cycle feedbacks. ISME J. 2, 805-814 (2008).

46. Hood-Nowotny, R., Umana, N. H.-N., Inselbacher, E., Oswald- Lachouani, P. \& Wanek, W. Alternative Methods for Measuring Inorganic, Organic, and Total Dissolved Nitrogen in Soil. Soil Sci. Soc. Am. J. 74, 1018-1027 (2010).

47. Torn, M. S. et al. A call for international soil experiment networks for studying, predicting, and managing global change impacts. SOIL Discuss. 2, 133-151 (2015).

48. Smith, P. et al. Interlinkages Between Desertification, Land Degradation, Food Security and Greenhouse Gas Fluxes: Synergies, Trade-offs and Integrated Response Options. in Climate Change and Land: an IPCC special report on climate change, desertification, land degradation, sustainable land management, food security, and greenhouse gas fluxes in terrestrial ecosystems (eds. Shukla et al.). (IPCC, 2019).

49. Angel, R., Claus, P. \& Conrad, R. Methanogenic archaea are globally ubiquitous in aerated soils and become active under wet anoxic conditions. ISME J. 6, 847-862 (2012).

50. Zhang, J., Kobert, K., Flouri, T. \& Stamatakis, A. PEAR: A fast and accurate Illumina Paired-End reAd mergeR. Bioinformatics 30, 614-620 (2014).

51. Kopylova, E., Noé, L. \& Touzet, H. SortMeRNA: Fast and accurate filtering of ribosomal RNAs in metatranscriptomic data. Bioinformatics 28, 3211-3217 (2012).

52. Schmieder, R. \& Edwards, R. Quality control and preprocessing of metagenomic datasets. Bioinformatics 27, 863-4 (2011).

53. NCBI Resource Coordinators. Database resources of the National Center for Biotechnology Information. Nucleic Acids Res. 46, D8-D13 (2018).

54. Buchfink, B., Xie, C. \& Huson, D. H. Fast and sensitive protein alignment using DIAMOND. Nat. Methods 12, 59-60 (2015). 
699 55. Huson, D. H. et al. MEGAN Community Edition - Interactive Exploration and Analysis of Large700 Scale Microbiome Sequencing Data. PLoS Comput. Biol. 12, 1-12 (2016).

701 56. R Core Development Team. A Language and Environment for Statistical Computing (R Foundation Statistical Computing, 2008).

703

57. Buttigieg, P. L. \& Ramette, A. A guide to statistical analysis in microbial ecology: A communityfocused, living review of multivariate data analyses. FEMS Microbiol. Ecol. 90, 543-550 (2014).

58. McDonald, J. H. Handbook of Biological Statistics (Sparky House Publishing, Baltimore, 2008). 


\section{Acknowledgements}

This study was supported by the Research Council of Norway FRIPRO Mobility Grant Project Time and Energy 251027/RU, co-founded by ERC under Marie Curie Grant 606895, and Troms $\varnothing$ Research Foundation starting grant project Cells in the Cold 16_SG_ATT. AR acknowledges funding by a JPI Climate Project (COUP-Austria, no. BMWFW- 6.020/0008). IJ and JP acknowledge the funding support from the European Research Council Synergy grant ERC-2013-SyG-610028 IMBALANCE-P. We thank Craig Herbold for assembling the transcripts and many intensive and fruitful discussions. Furthermore, we thank Petra Pjevac for discussions, Tilman Schmider for contributing to the figure designs, and Thomas Rattei and Florian Goldenberg for bioinformatics support.

\section{Author contributions}

AR, ATT, TU, and AS conceived the study. BDS, AR, IJ, and JPe established the experimental sites. AR and JPr collected the samples. ATT, JPr, and JS processed the samples in the lab. AS, ATT, and MBD analysed the sequencing data. AS created the figures. AS and ATT wrote the manuscript with inputs from all authors.

\section{Competing interests}

The authors declare no competing interests.

\section{Materials and correspondence}

Corresponding authors: Alexander T. Tveit (alexander.t.tveit@uit.no) and Andrea Söllinger (andrea.soellinger@uit.no).

\section{Additional information}

Supplementary information, figures, and tables are available. The raw sequence data are available at the NCBI Sequence Read Archive (SRA); BioProject ID: PRJNA663238, accession numbers SAMN16124403- SAMN16124422. 


\section{Figures}

a

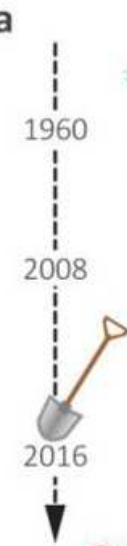

(0) Earthquake

Soil sampling \& metatranscriptomics b

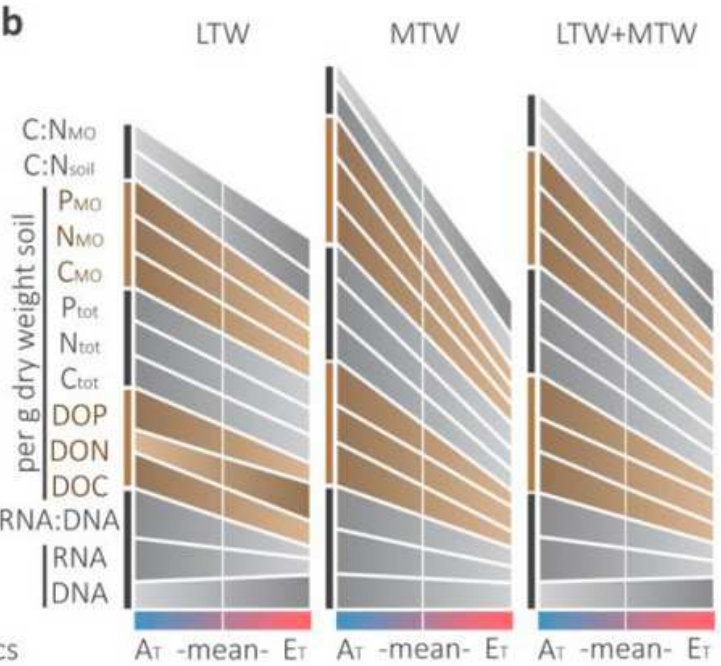

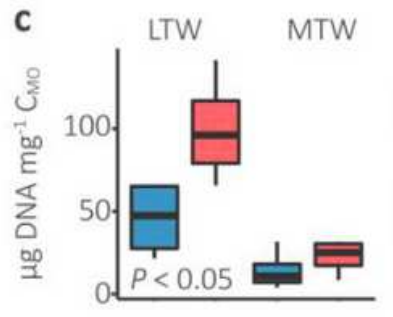
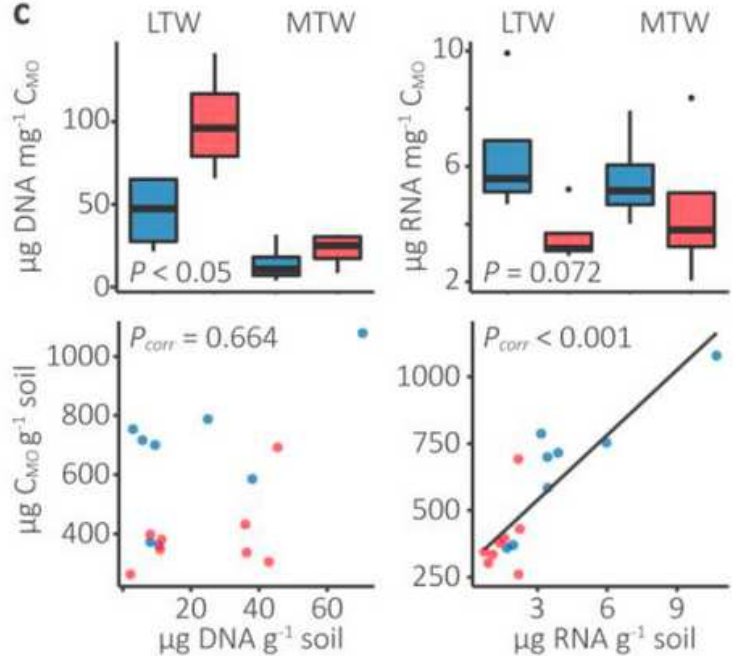

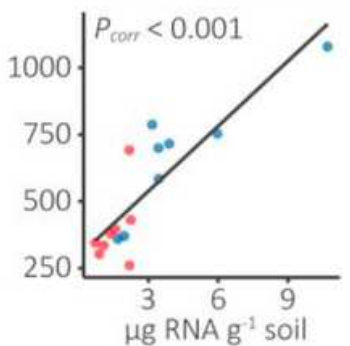

Figure 1

Grassland samples and warming-induced differences on physicochemical and biological properties. a Schematic representation of the study sites, their development over time, and the metatranscriptomics samples (see Methods for details). b Trend charts (see Methods) indicating differences in DNA and RNA concentrations (per unit of soil), contents of dissolved organic $C, N$, and $P$ (DOC, DON, and DOP, respectively), total $\mathrm{C}, \mathrm{N}$, and $\mathrm{P}$ (Ctot, Ntot, and Ptot, respectively), microbial $\mathrm{C}, \mathrm{N}$, and $\mathrm{P}$ (CMO, NMO, and PMO, respectively), RNA:DNA ratio, and soil and microbial C:N ratios (see Supplementary Tables 1 and 2 for absolute values and significant differences, respectively). c DNA and RNA content per unit of microbial biomass and correlations between microbial biomass and DNA and RNA content per unit of soil (Supplementary Table 3). 
a

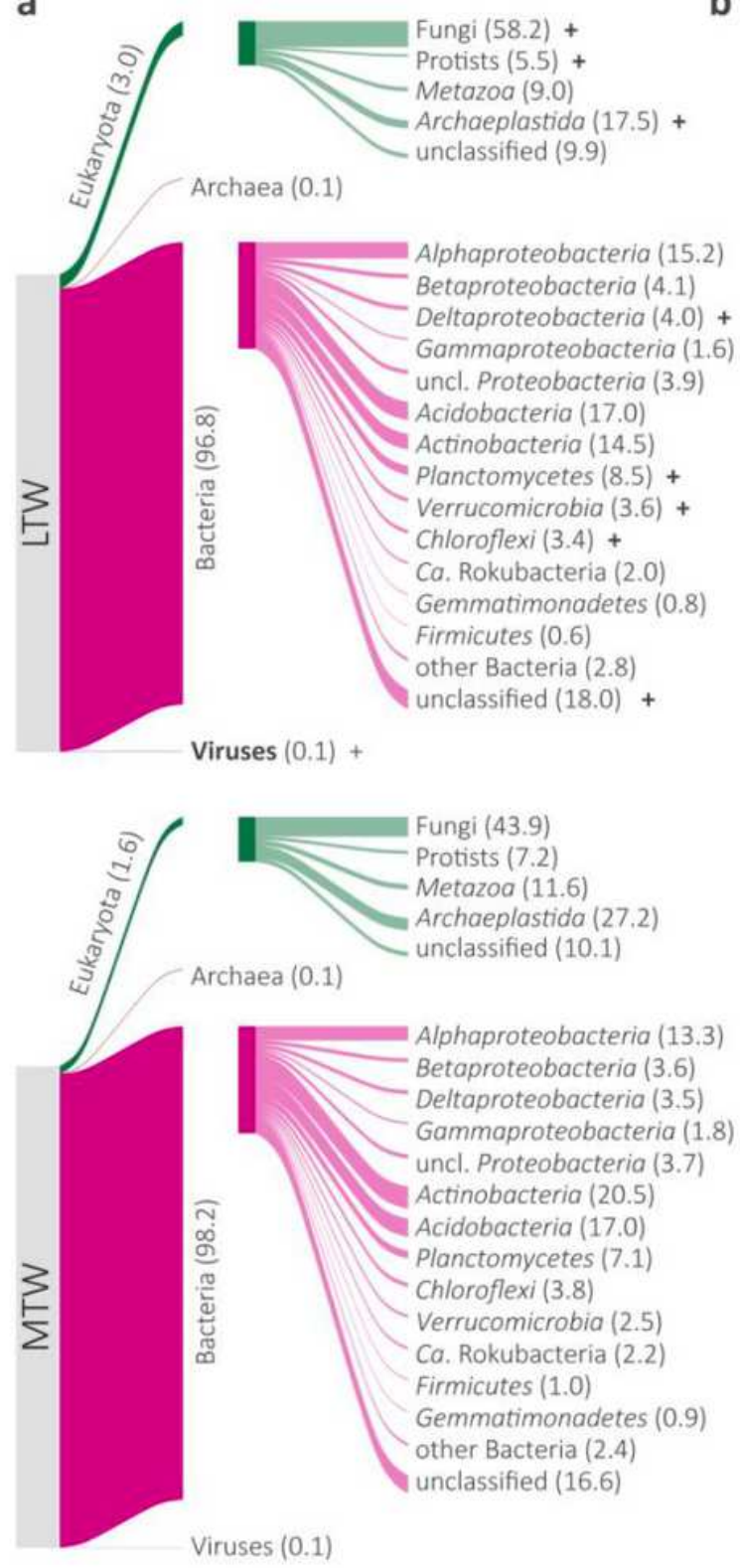

b

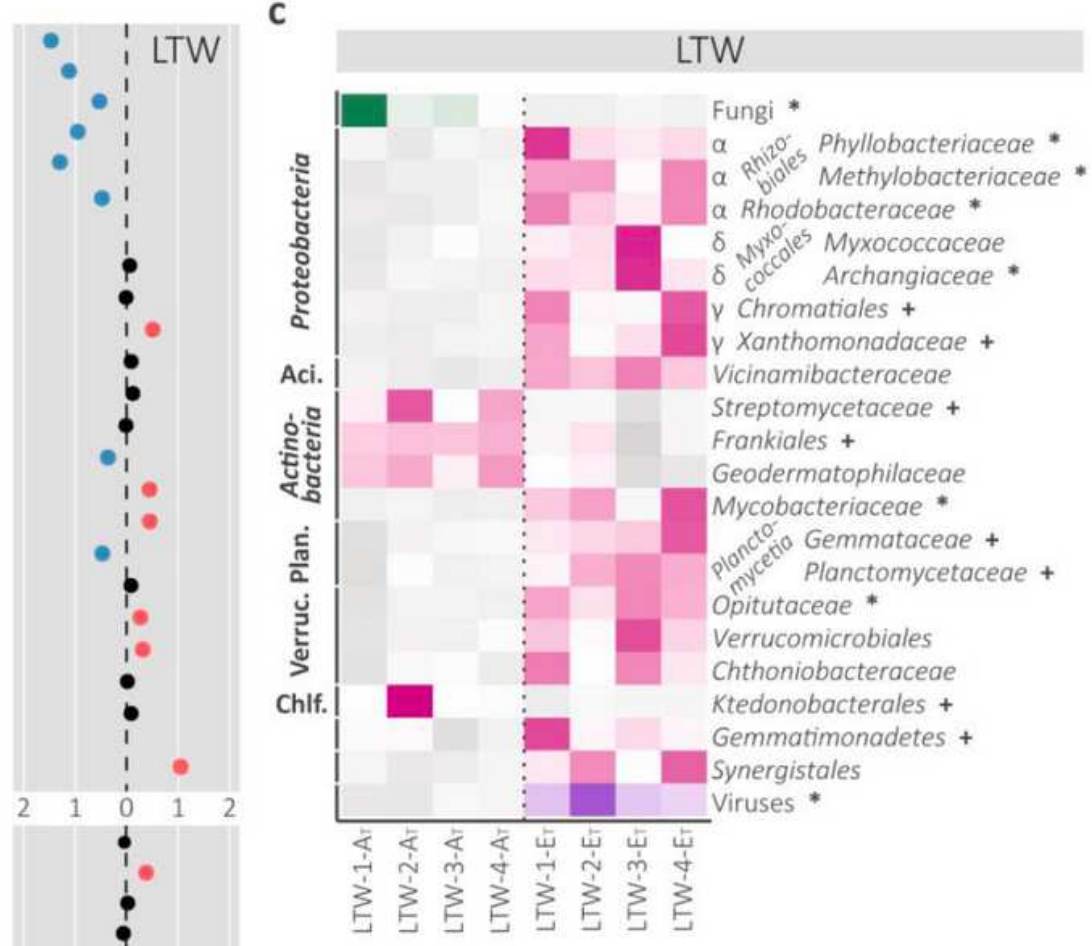

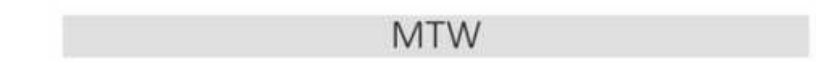

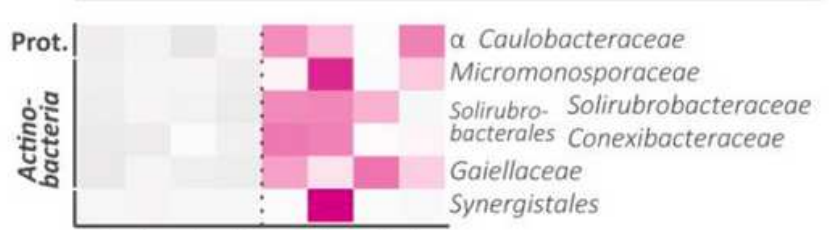

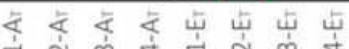

-1

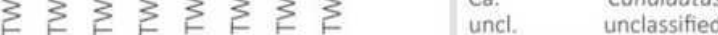
a Alphaproteobacteria y Gammaproteobacteria $\delta$ Deltaproteobacteria Aci. Acidobacteria Plan. Planctomycetes Verruc. Verrucomicrobia Chlf. Chloroflexi Prot. Proteobacteria

\section{Figure 2}

Taxonomic annotation of mRNA reads and warming-induced abundance patterns. a Sankey plots showing both the fraction of mRNA reads (mean over all LTW and MTW replicates, respectively) assigned to domains and the composition and relative abundances of eukaryotic and bacterial mRNAs. The depicted groups account for $100 \%$ of eukaryotic and bacterial mRNAs, respectively. All bacterial phyla with a relative abundance of $\geq 1 \%$ in at least one of the sampled soil groups are depicted; the remaining phyla are summed (other Bacteria). Potential warming-induced differences in mRNA abundances are indicated with + (t-tests, $n=8, P<0.05$, Pcorr < 0.1; Supplementary Table 6). b Log2-fold changes of mean relative abundances between AT and ET of the taxa listed left-hand. c Exploratory analysis showing warming-induced taxon abundance patterns. Only taxa with higher or lower relative abundances in all four replicates of one group relative to their counterparts are depicted (4/4-filter, see Methods). Bacterial 
taxa are shown at family level; higher taxonomic levels are only shown if no family belonging to these higher levels passed the filter. Higher bacterial taxonomic levels are accordingly not shown if any family belonging to these levels passed the filter. Potential warming-induced differences in mRNA abundances are indicated (differential gene expression analysis, $n=8$, Pcorr $<0.05(*)$, Pcorr $<0.1(+)$ ); Supplementary Table 7). We subsequently analysed functional mRNA annotations to identify how soil warming influences transcription of genes involved in central metabolic functions and cellular processes.

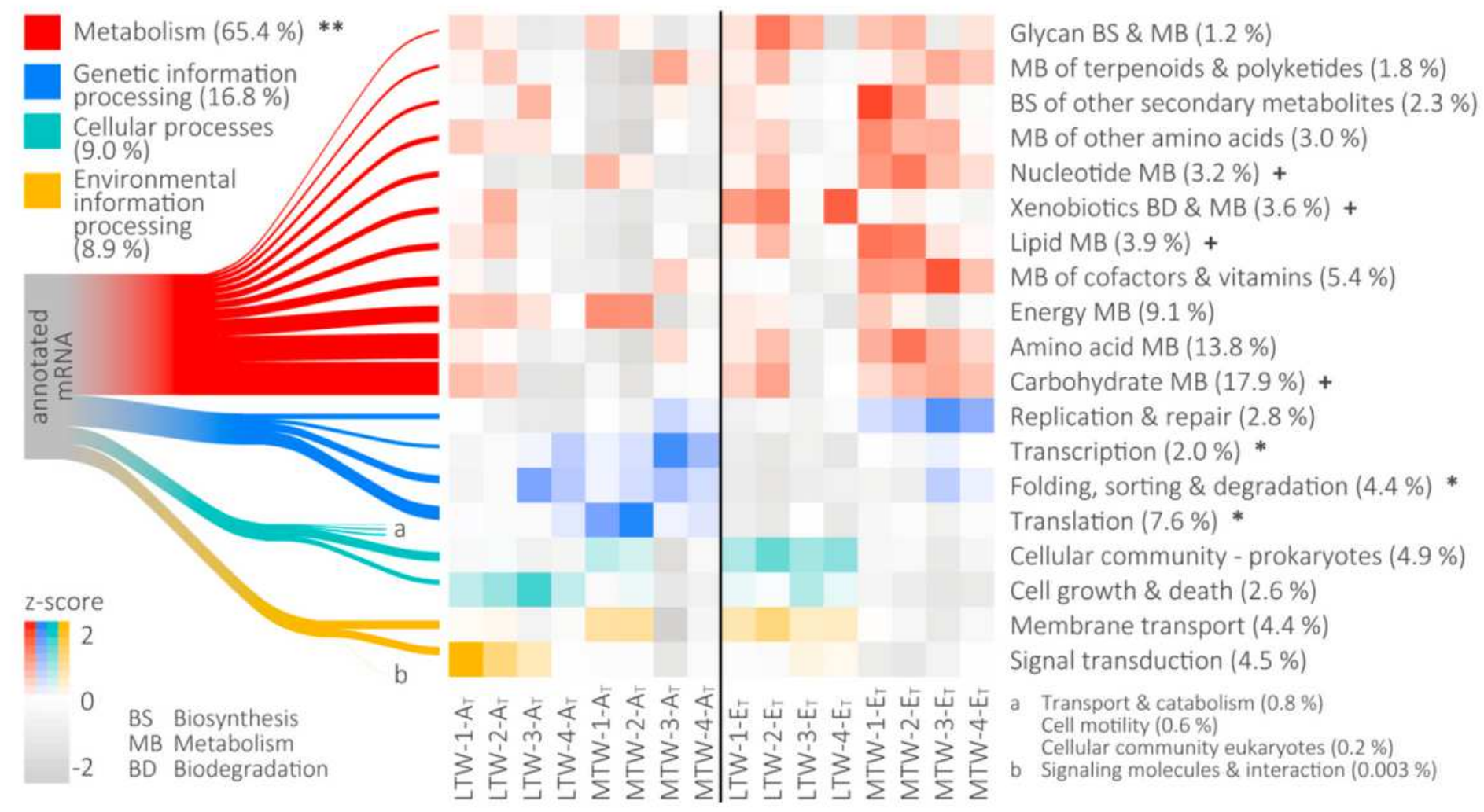

\section{Figure 3}

Functional annotation of mRNA reads. Sankey diagram showing the mean relative abundances of KEGG1 and KEGG2 categories over all samples. The heatmap depicts the relative abundances of all KEGG2 categories with mean relative abundances $>1 \%$; samples are sorted by soil temperature (AT, ambient soil temperature; $\mathrm{ET},+6^{\circ} \mathrm{C}$ ). The heatmap colour code indicates the KEGG1 affiliation of the KEGG2 categories. Potential warming-induced differences in mRNA abundances are indicated (differential gene expression analysis, $n=16$, Pcorr $<0.01(* *)$, Pcorr $<0.05(*)$, Pcorr $<0.1(+)$; SupplementaryTable 8). 


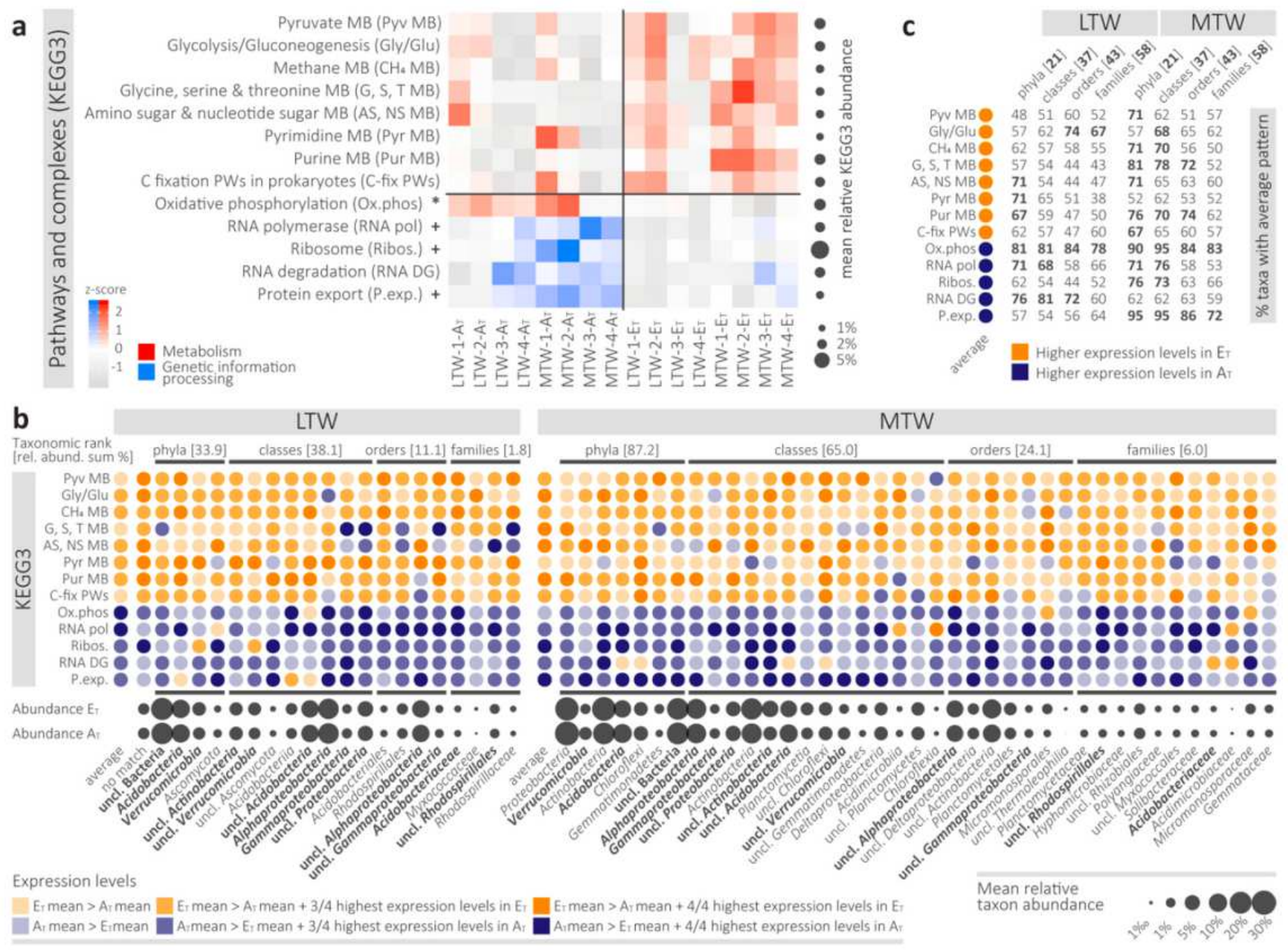

\section{Figure 4}

Overall and taxon-specific warming-induced transcript abundance patterns. a Pathways and complexes with mean relative abundances $\geq 1 \%$ that passed the differential abundance-pattern filter (13/16-filter, see Methods). Colour code indicates the corresponding KEGG1 category. Differential gene expression analysis results are indicated next to the KEGG 3 categories $(n=16$, Pcorr $<0.05(*)$, Pcorr $<0.1(+)$; Supplementary Table 8). b Taxa reflecting the observed warming-induced abundance patterns presented in (a). All taxa with a mean relative abundance $\geq 1 \%$ in LTW-AT, LTW-ET, MTW-AT, or MTW-ET and not more than two deviations from the overall pattern are depicted. Numbers in square brackets next to the taxonomic ranks are the sums of the relative abundances of the individual taxa represented below (grey circles). Colour code indicates the mean relative abundance of a KEGG3 category: orange, higher expression levels in ET; blue, higher expression levels in AT. Pale, dark, and intermediate colours indicate how strong and widely distributed a pattern was across LTW and MTW soils, respectively (see legend "Expression levels"). See (a) for KEGG3 abbreviations. c Table listing the percentage of all abundant taxa (i.e. taxa with $>1 \%$ relative abundance) within a taxonomic rank that featured the overall warminginduced abundance pattern of a specific KEGG3 category (see Supplementary Fig. 6 for details on the 
taxa). Numbers in square brackets next to the taxonomic ranks give the total number of abundant taxa within the taxonomic rank. Bold numbers indicate pathways and complexes with warming-induced differential abundance patters observed in more than two thirds of all abundant taxa within a taxonomic rank.

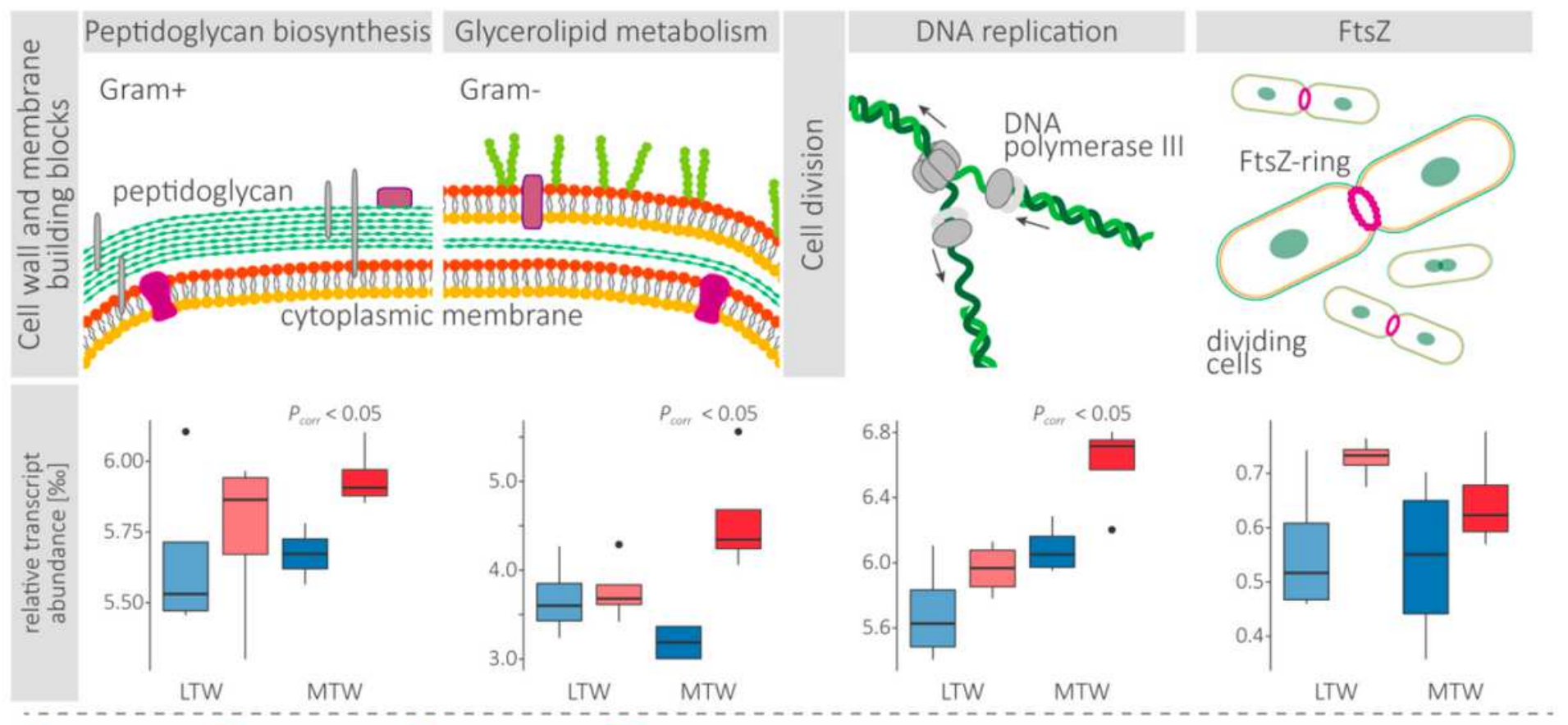

$\operatorname{LTW}-\mathrm{AT}_{T}\left(+0^{\circ} \mathrm{C}\right) \square \operatorname{LTW}-\mathrm{ET}_{\mathrm{T}}\left(+6^{\circ} \mathrm{C}\right) \square \operatorname{MTW}-\mathrm{AT}_{\mathrm{T}}\left(+0^{\circ} \mathrm{C}\right) \square \operatorname{MTW}-\mathrm{ET}_{\mathrm{T}}\left(+6^{\circ} \mathrm{C}\right)$

\section{Figure 5}

Warming-induced abundance profiles of bacterial growth-related transcripts. Boxplots showing relative transcript abundances of the KEGG3 categories Peptidoglycan biosynthesis, Glycerolipid metabolism, and DNA replication, and FtsZ in the bacterial fractions of the LTW-AT, LTW-ET, LTW-AT, and LTW-ET metatranscriptomes. The depicted KEGG3 categories are involved in the build-up of new cell walls and cell membranes and responsible for the duplication of genomic DNA, which precedes bacterial cell division; and FtsZ represents a key enzyme in bacterial cell division (see schematic drawings above the boxplots). P-values (Pcorr) indicating significant differences are displayed above boxplot-pairs (Supplementary Table 9). 


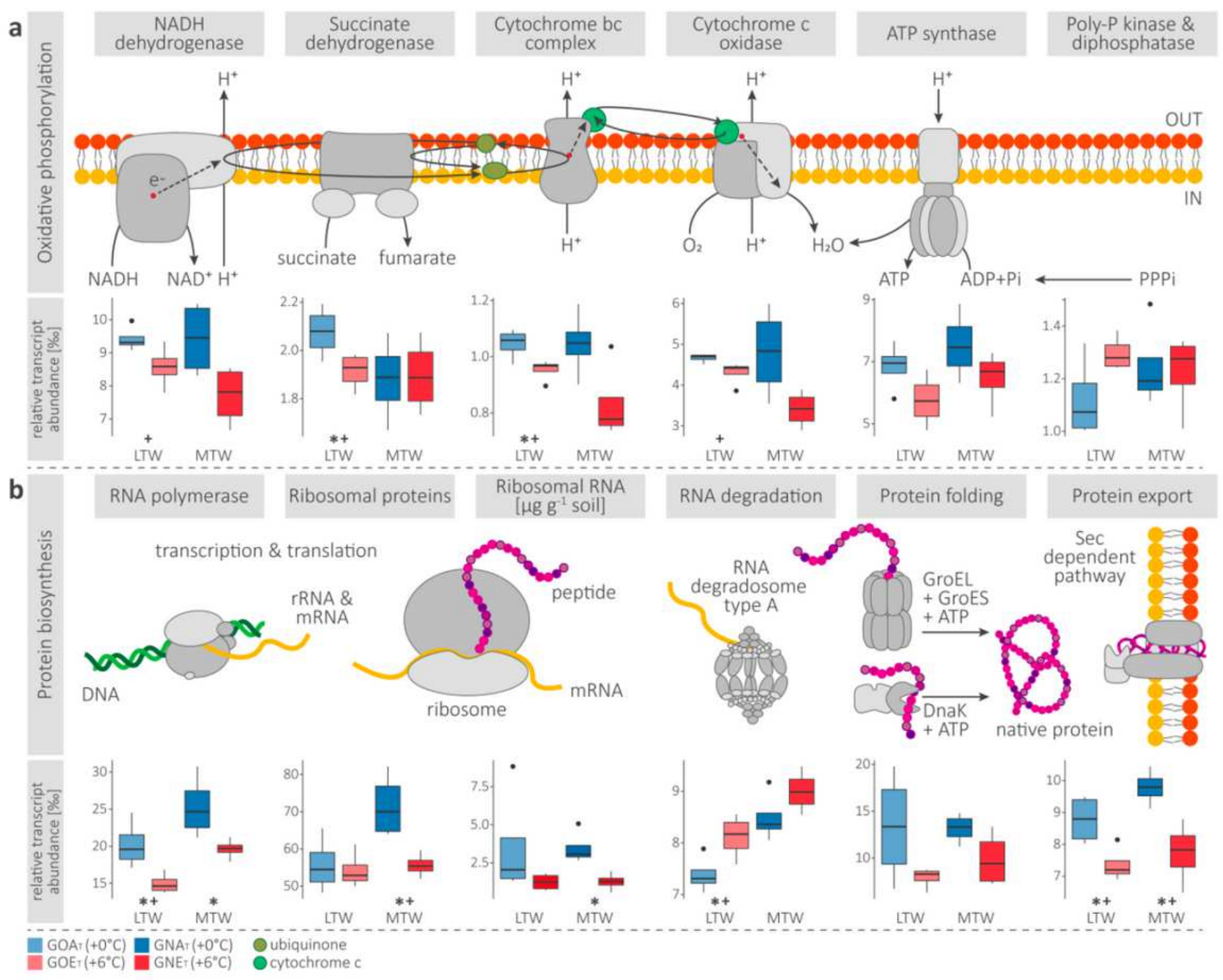

Figure 6

Warming-induced abundance profiles of transcripts related to bacterial energy metabolism and protein biosynthesis. Boxplots show the relative transcript abundances of enzymes and enzyme complexes involved in (a) membrane-bound electron transport and ATP synthesis (oxidative phosphorylation) and (b) protein biosynthesis in the LTW-AT, LTW-ET, LTW-AT, and LTW-ET metatranscriptomes (see Supplementary Data 1 for a list of KOs summarised in the boxplots). Schematic representations of the enzymes and enzyme complexes are provided above each boxplot and are based on the KEGG pathway drawings. Membrane-bound complexes are embedded in a lipid bilayer. Potential warming-induced differences are indicated by $\mathrm{p}$-values (uncorrected) $<0.05\left(^{*}\right)$ and corrected p-values $<0.1(+)$ (t-tests, $\mathrm{n}=$ 8, Supplementary Table 10). GroEL and DnaK are part of the KEGG3 category RNA degradation, thus skewing its overall warming-induced abundance pattern towards lower transcript abundances in ET than AT as seen in (Fig. 4a). 


\section{Supplementary Files}

This is a list of supplementary files associated with this preprint. Click to download.

- SupplementarydataS1.xlsx

- SupplementarydataS2.xlsx

- Soellingeretalforhotsupplementfinal.pdf 